\title{
On the Doubly Refined Enumeration of Alternating Sign Matrices and Totally Symmetric Self-Complementary Plane Partitions
}

\author{
Tiago Fonseca* \\ LPTHE (CNRS, UMR 7589), Univ Pierre et Marie Curie-Paris6, \\ 75252 Paris Cedex, France \\ fonseca @ lpthe.jussieu.fr \\ Paul Zinn-Justin ${ }^{\dagger}$ \\ LPTMS (CNRS, UMR 8626), Univ Paris-Sud, \\ 91405 Orsay Cedex, France; \\ and LPTHE (CNRS, UMR 7589), Univ Pierre et Marie Curie-Paris6, \\ 75252 Paris Cedex, France \\ pzinn @lpthe.jussieu.fr
}

Submitted: Mar 26, 2008; Accepted: Jun 5, 2008; Published: Jun 13, 2008

\begin{abstract}
We prove the equality of doubly refined enumerations of Alternating Sign Matrices and of Totally Symmetric Self-Complementary Plane Partitions using integral formulae originating from certain solutions of the quantum KnizhnikZamolodchikov equation.
\end{abstract}

${ }^{*}$ The authors thank N. Kitanine for discussions, and J.-B. Zuber for a careful reading of the manuscript.

${ }^{\dagger}$ PZJ was supported by EU Marie Curie Research Training Networks "ENRAGE" MRTNCT-2004-005616, "ENIGMA" MRT-CT-2004-5652, ESF program "MISGAM" and ANR program "GIMP" ANR-05-BLAN-0029-01. 


\section{Contents}

1 Introduction 2

2 The models 3

2.1 Alternating Sign Matrices . . . . . . . . . . . . . . . . . . . . . . . . 3

2.2 6-Vertex model . . . . . . . . . . . . . . . . . . . . . . . . 4 4

2.3 Totally Symmetric Self-Complementary Plane Partitions . . . . . . . . . . . . . . . . 4

2.4 Non-Intersecting Lattice Paths . . . . . . . . . . . . . . . . . . . . . . 6

3 The conjecture $\quad 8$

3.1 ASM generating function $\ldots \ldots \ldots \ldots \ldots \ldots \ldots$

3.2 NILP generating function $\ldots \ldots \ldots \ldots \ldots$

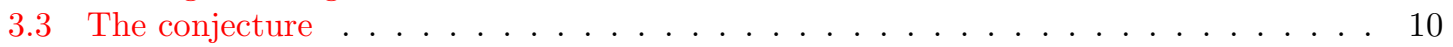

4 The proof $\quad 10$

4.1 ASM counting as the partition function of the 6 -Vertex model . . . . . . . . . . 10

4.2 Integral formula for refined ASM counting . . . . . . . . . . . . . . 13

4.3 Integral formula for refined NILP counting . . . . . . . . . . . . . . . . 16

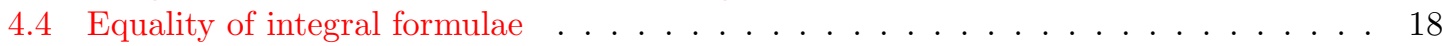

A Formulating the conjecture directly in terms of TSSCPPs $\quad 20$

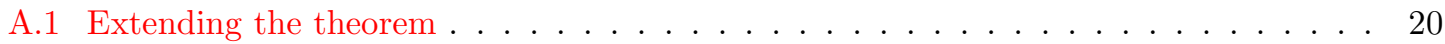

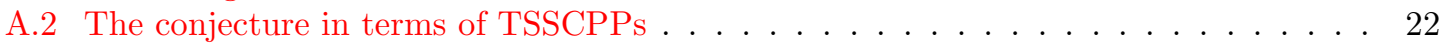

B Properties of the 6-Vertex model partitionfunction 23

B.1 Korepin recursion relation . . . . . . . . . . . . . . . . . . . . . 24

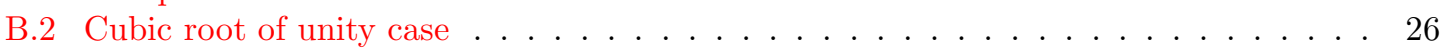

$\begin{array}{ll}\text { C The space of polynomials satisfying the wheel condition } & 27\end{array}$

D An antisymmetrization formula $\quad 29$

D.1 The general case . . . . . . . . . . . . . . . . . . . . 29

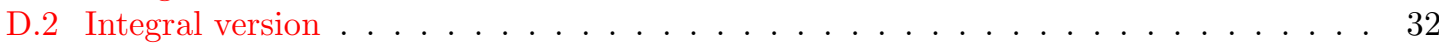

D.3 Homogeneous Limit . . . . . . . . . . . . . . . . . . . . . . 33

\section{Introduction}

It is the purpose of this work to revisit an old problem using some new ideas. The old problem is the interconnection between two distinct classes of combinatorial objects whose enumerative properties are intimately related: Alternating Sign Matrices and Plane Partitions [2]. The new ideas come from recent developments in the so-called Razumov-Stroganov conjecture (formulated in [19]; see also [1, 3]). The RazumovStroganov conjecture identifies the entries of the Perron-Frobenius vector of a certain 
stochastic matrix with cardinalities of subsets of Alternating Sign Matrices, the latter being reinterpreted as configurations of a certain two-dimensional statistical model (so-called Fully Packed Loops). Even though this statement is still a conjecture, some progress has been made in this area in a series of papers by Di Francesco and ZinnJustin, starting with [4]. The method they used was, as it turned out, equivalent to finding appropriate polynomial solutions of the quantum Knizhnik-Zamolodchikov equation [5]. Integral representations for these and their relation to plane partition enumeration were discussed in [6]; we shall use these integral formulae in the present work (noting that these can be considered as purely formal integrals, so they are simply a way of encoding generating functions).

The paper is organized as follows. In section 2, we define the various combinatorial objects and corresponding statistical models that will be needed. In section 3, we formulate the main theorem of the paper: the equality of doubly refined enumerations of Alternating Sign Matrices and of Totally Symmetric Self-Complementary Plane Partitions. Section 4 contains the proof, based on the use of integral formulae. Finally, the appendices contain various technical results that are needed in the proof. Note that even though we use some concepts and methods from exactly solvable statistical models, this paper is self-contained and all proofs are purely combinatorial in nature.

\section{The models}

In this section we define the various models that appear in this work. There are two distinct models. On the one hand we have Alternating Sign Matrices (ASMs) which are in bijection with configurations of the 6-Vertex model (also known as ice model) with Domain Wall Boundary Conditions, as well as with Fully Packed Loop configurations (FPL). Here we only discuss ASMs and 6-V model.

On the other hand we have Totally Symmetric Self-Complementary Plane Partitions, which are in bijection with a certain class of Non-Intersecting Lattice Paths.

\subsection{Alternating Sign Matrices}

An Alternating Sign Matrix (ASM) is a square matrix made of 0s, 1s and -1s such that if one ignores $0 \mathrm{~s}, 1 \mathrm{~s}$ and $-1 \mathrm{~s}$ alternate on each row and column starting and ending with 1 s. Here are all $3 \times 3$ ASMs:

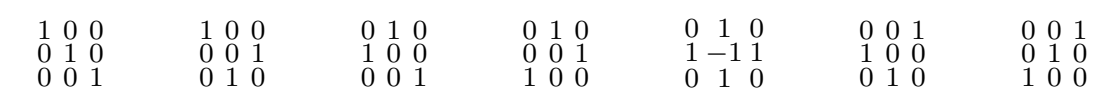




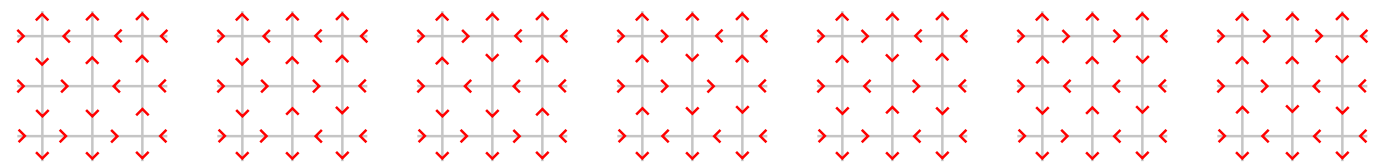

Figure 1: The 6-Vertex Model is defined on a $n \times n$ grid. To each link in the network we associate an arrow which can take two directions, the only constraint being that at each site there are two arrows pointing in and two arrows pointing out (this leaves 6 possible vertex configurations). We are only interested in the configurations such that the arrows at the top and at the bottom are pointing out and the arrows at the left and the right are pointing in. Here we draw all states possibles for $n=3$.

Thus, there are exactly 7 ASMs of size $n=3$.

These matrices have been studied by Mills, Robbins and Rumsey since the early 1980s $[14,15,21,16]$. It was then conjectured that $A_{n}$, the number of ASMs of size $n$, is given by:

$$
A_{n}=\prod_{j=0}^{n-1} \frac{(3 j+1) !}{(n+j) !}=1,2,7,42,429, \ldots
$$

This was subsequently proved by Zeilberger in 1996 in an 84 page article [23]. A shorter proof was given by Kuperberg [12] in 1998. The latter is based on the equivalence to the 6 - $\mathrm{V}$ model, which we shall also use here.

\subsection{6 -Vertex model}

Let us now turn to the 6 -Vertex Model. The model consists in a square grid of size $n \times n$ in which each edge is given an orientation (an arrow), such that at each vertex there are two arrows pointing in and two arrows pointing out. We use here some very specific boundary conditions (Domain Wall Boundary Conditions, DWBC): all arrows at the left and the right are pointing in and at the bottom and the top are pointing out.

On figure 1 we draw all the possible configurations at $n=3$. There are once again 7 configurations of size $n=3$. Indeed, there is an easy bijection between ASMs and 6-V configurations with DWBC, which is described schematically on figure 2.

\subsection{Totally Symmetric Self-Complementary Plane Partitions}

We describe here Plane Partitions in two different ways, either pictorially or as arrays of numbers. 


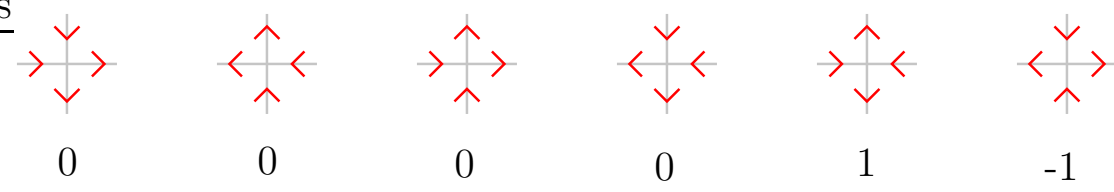

Figure 2: Rules to replace each vertex of a $6-\mathrm{V}$ configuration with a 0 or \pm 1 . Conversely, one can consistently build a $6-\mathrm{V}$ configuration from an ASM starting from the fixed arrows on the boundary, continuing arrows through the $0 s$ and reversing them through the \pm 1 .

Pictorially, a plane partition is a stack of unit cubes pushed into a corner (gravity pushing them to the corner) and drawn in isometric perspective, as examplified on figure 3.

An equivalent way of describing these objects is to form the array of heights of each stack of cubes. In this formulation the effect of "gravity" is that each number in the array is less or equal than the numbers immediately above and to the left. For example the plane partition on figure 3 may be translated into the array

Plane partitions were first introduced by MacMahon in 1897. A problem of interest is the enumeration of plane partitions that have some specific symmetries. The Totally Symmetric and Self-Complementary Plane Partitions (TSSCPPs) are one of these symmetry classes. In the pictorial representation, they are Plane Partitions inside a $2 n \times 2 n \times 2 n$ cube which are invariant under the following symmetries: all permutations of the axes of the cube of size $2 n \times 2 n \times 2 n$; and taking the complement, that is putting cubes where they are absent and vice versa, and flipping the resulting set of cubes to form again a Plane Partition.

Alternatively, they can be described as $2 n \times 2 n$ arrays of heights. In the $n=3$ 


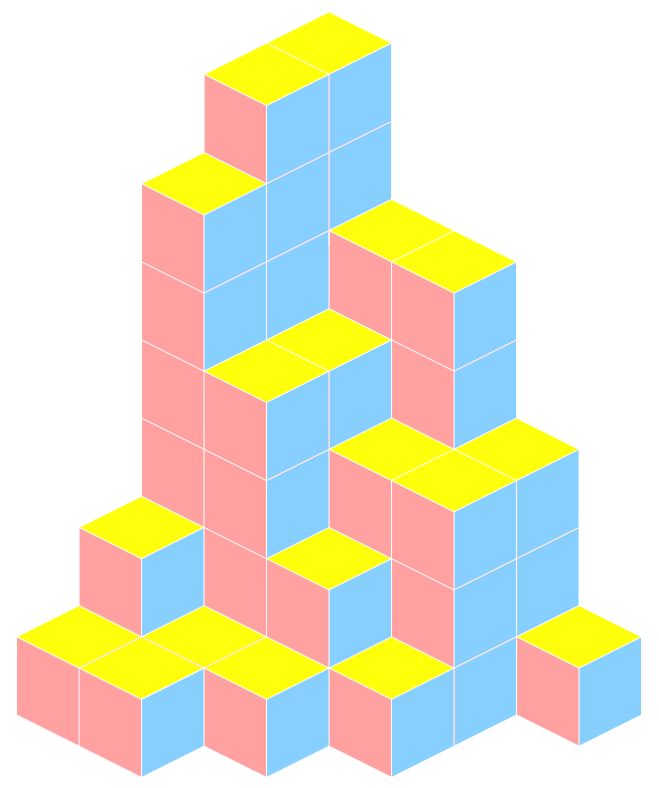

Figure 3: We can see a plane partition (PP) as a stack of unit cubes pushed into a corner.

case, we have, once again, 7 possible configurations:

$\begin{array}{lllllll}666333 & 666433 & 666433 & 666543 & 666543 & 666553 & 666553 \\ 666333 & 666333 & 666433 & 665332 & 665432 & 655331 & 655431 \\ 666333 & 665332 & 664322 & 655331 & 654321 & 655331 & 654321 \\ 333000 & 433100 & 443200 & 533110 & 543210 & 533110 & 543210 \\ 333000 & 333000 & 332000 & 433100 & 432100 & 533110 & 532110 \\ 333000 & 332000 & 332000 & 321000 & 321000 & 311000 & 311000\end{array}$

and more generally we obtain $A_{n}$ for any $n$. In fact Zeilberger's proof of the ASM conjecture amounts to showing (non-bijectively) that ASMs and TSSCPPs are equinumerous.

\subsection{Non-Intersecting Lattice Paths}

Another important class of objects is the Non-Intersecting Lattice Paths (NILPs). These paths are defined in a lattice and connect a set of initial points to a set of final points following certain rules (see Ref. [13, 7] for the general framework). The most important feature of NILPs is that the various paths do not touch one another. 


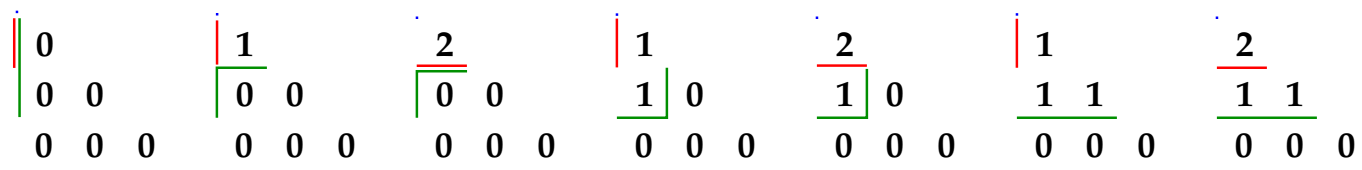

Figure 4: Reformulation of TSSCPPs as NCLPs, in the example of size $n=3$. If the origin is at the upper right corner, then at each point $(0,-i), i \in\{0, \ldots, n-1\}$, begins a path which can only go upwards or to the right, and stops when it reaches the diagonal $(j,-j)$, in such a way that the numbers below/to the right of it are exactly those less or equal to $n-i$.

In order to better understand the bijection between NILPs and TSSCPPs, it is convenient to consider an intermediate class of objects: Non-Crossing Lattice Paths (NCLPs), which are similar to NILPs except for the fact the paths are allowed to share a common site, although they are still forbidden to cross each other.

We proceed with the description of the bijection between TSSCPPS and a class of NCLPs. Each TSSCPP is defined by a subset of numbers of the arrays of (2.2), a possible choice is the triangles at the bottom right:

$\begin{array}{lllllll}0 & 1 & 2 & 1 & 2 & 1 & 2 \\ 00 & 00 & 00 & 10 & 10 & 11 & 11 \\ 000 & 000 & 000 & 000 & 000 & 000 & 000\end{array}$

It is easy to prove that this part of the array together with the symmetries which characterize the TSSCPPs are enough to reconstruct the whole TSSCPP.

Then, we draw paths separating the different numbers appearing, as explained on figure 4.

The bijection with the NILPs is easily achieved by shifting the paths (NCLPs) according to the following rules:

- The $i^{\text {th }}$ path begins at $(i,-i)$;

- The vertical steps are conserved and the horizontal steps $(\rightarrow)$ are replaced by diagonal steps $(\nearrow)$.

An example $(n=3)$ is shown on figure 5 .

Our last modification is the addition of one extra step to all paths. To the first path we add a diagonal step, as for the other paths the choice is made such that the difference between the final point of two consecutive paths is an odd number, as examplified on figure 6 . 


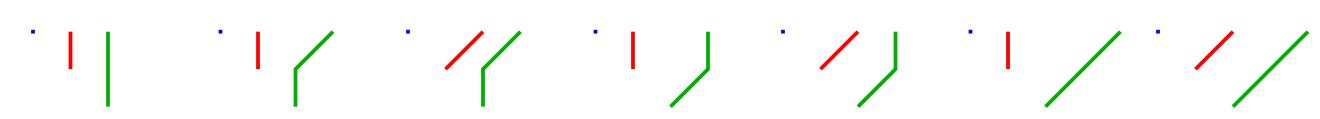

Figure 5: We transform our NCLPs into NILPs: the starting point is now shifted to the right, and the horizontals steps become diagonal steps.

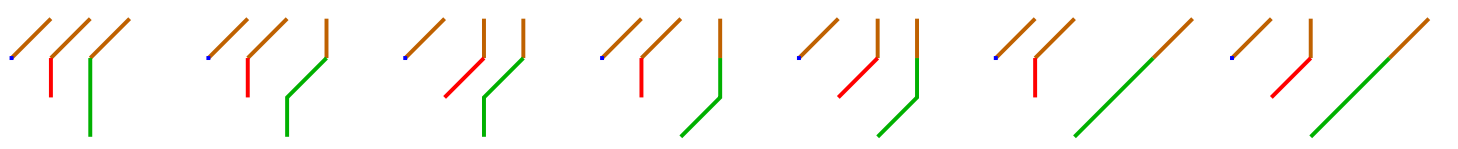

Figure 6: To each path we add one extra step in order that two final points consecutive differ by an odd number. The first extra step is diagonal.

\section{The conjecture}

Various conjectures have been made to connect ASMs and TSSCPPs. Building on the already mentioned ASM conjecture by Mills and Robbins, which says that the number of ASMs of size $n$ is equal to the number of TSSCPPs of size $2 n$ (and which is now a theorem), there are conjectures about "refined" enumeration. Before describing them we need some more definitions.

\subsection{ASM generating function}

Each ASM, as can be easily proven, has one and only one 1 on the first row and on the last row. It is natural to classify ASMs according to their position. Therefore, we count the ASMs of size $n$ with the first 1 in the $i^{\text {th }}$ position and the last 1 in the $j^{\text {th }}$ position: $\tilde{A}_{n, i, j}$.

We build the corresponding generating function:

$$
\tilde{A}_{n}(x, y):=\sum_{i, j} \tilde{A}_{n, i, j} x^{i-1} y^{j-1}
$$

We define also $A_{n, i, j}$, which counts the ASMs with the first 1 in the $i^{\text {th }}$ column and the last 1 in the $(n-j+1)^{\text {st }}$ column:

$$
A_{n, i, j}=\tilde{A}_{n, i, n-j+1}
$$

And its generating function:

$$
A_{n}(x, y):=\sum_{i, j} A_{n, i, j} x^{i-1} y^{j-1}
$$




\section{Some trivial symmetries.}

By reflecting the ASMs horizontally and vertically one gets:

$$
A_{n, i, j}=A_{n, j, i}
$$

whereas by reflecting them only horizontally one gets:

$$
A_{n, i, j}=A_{n, n-i+1, n-j+1}
$$

Obviously these symmetries are also valid for $\tilde{A}_{n, i, j}$.

\subsection{NILP generating function}

First we recall the definition of the type of NILPs used in this article, of size $n$ :

- The paths are defined on the square grid. Each step connects a site to a neighbor and can be either vertical (up $\uparrow$ ) or diagonal (up right $\nearrow$ ).

- There are $n$ starting points with coordinates $(i,-i), i \in\{0,1, \ldots, n-1\}$. The endpoints are at $(i, 0)$ (so that the length of the $i^{\text {th }}$ path is $i$ ).

- Paths do not touch each other.

It is convenient to add an extra step, as explained in section 2.4, defined uniquely by the following:

- Two consecutive paths, after the extra step, differ by an odd number.

- The extra step for the first path $($ at $(0,0))$ is diagonal.

Let $\alpha$ be a NILP, we define $u_{n}^{0}(\alpha)$ as the number of vertical steps in the extra step and $u_{n}^{1}(\alpha)$ as the number of vertical step in the last step of each path (see appendix A.1 for an extended definition).

The generating function is:

$$
U_{n}^{0,1}(x, y):=\sum_{\alpha} x^{u^{0}(\alpha)} y^{u^{1}(\alpha)}=\sum_{i, j} U_{n, i, j}^{0,1} x^{i} y^{j}
$$

where $U_{n, i, j}^{0,1}$ is the number of NILPs of size $n$ with $i$ vertical extra steps and $j$ vertical last steps. 


\subsection{The conjecture}

We now present the conjecture, formulated by Mills, Robbins and Rumsey in a slightly different language (see section A.2 for a detailed translation), whose proof is the main focus of the present work:

Theorem. The number of ASMs of size $n$ with the 1 of the first row in the $(i+1)^{\mathrm{st}}$ position and the 1 of last row in the $(j+1)^{\text {st }}$ position is the same as the number of NILPs (corresponding to TSSCPPs, and with the extra step) with $i$ vertical extra steps and $j$ vertical steps in the last step. Equivalently,

$$
\tilde{A}_{n}(x, y)=U_{n}^{0,1}(x, y)
$$

For example, at $n=3$, using the ASMs given in section 2.1 and the TSSCPPs given on figure 6 , we compute:

$$
\begin{gathered}
\tilde{A}_{3}(x, y)=y^{2}+y+x y^{2}+x+x y+x^{2} y+x^{2} \\
U_{n}^{0,1}(x, y)=y^{2}+x y+x^{2}+x y^{2}+x^{2} y+y+x
\end{gathered}
$$

This is the doubly refined enumeration. Of course, by specializing one variable, one recovers the simple refined enumeration, i.e. that the number of ASMs of size $n$ with the 1 of the first row in the $i+1$ position is the same as the number of NILPs (corresponding to the TSSCPPs and with the extra step) with $i$ vertical extra steps:

$$
A_{n}(x):=\tilde{A}_{n}(x, 1)=U_{n}^{0,1}(x, 1):=U_{n}^{0}(x)
$$

and by specializing two variables, that the number of ASMs of size $n$ is the same as the number of TSSCPPs of size $2 n$ :

$$
A_{n}=A_{n}(1)=U_{n}^{0}(1)
$$

\section{The proof}

\subsection{ASM counting as the partition function of the 6-Vertex model}

In order to solve the ASM enumeration problem, it is convenient to generalize it by considering weighted enumeration. This amounts to computing the partition function of the 6-Vertex model, that is the summation over 6-V configurations with DWBC such that to each vertex is given a statistical weight, as shown on figure 7 , 


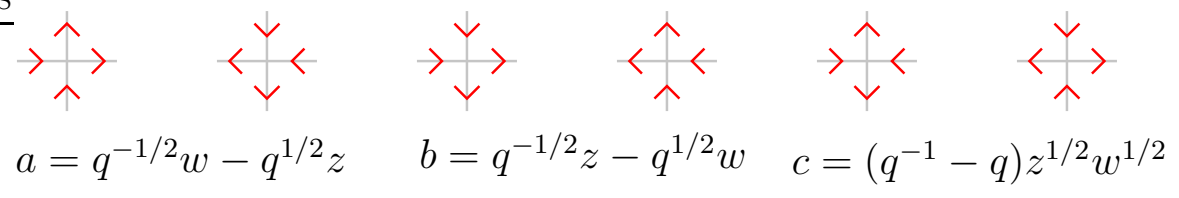

Figure 7: To each site configuration corresponds a statistical weight. These weights depend on three parameters: $w$ (resp. $z$ ) which characterizes the column (resp. row), and a global parameter $q$ which will be eventually specialized to a cubic root of unity.

depending on $n$ horizontal spectral parameters (one for each row) $\left\{z_{1}, z_{2}, \ldots, z_{n}\right\}, n$ vertical spectral parameters $\left\{z_{n+1}, z_{n+2}, \ldots, z_{2 n}\right\}$ and one global parameter $q$. This computation was performed by Izergin [8], using recursion relations written by Korepin [11], and the result is a $n \times n$ determinant (IK determinant). It is a symmetric function of the set $\left\{z_{1}, \ldots z_{n}\right\}$ and of the set of $\left\{z_{n+1}, \ldots, z_{2 n}\right\}$. Much later, it was observed by Stroganov [22] and Okada [17] that when $q=e^{2 \pi i / 3}$, the partition function is totally symmetric, i.e. in the full set $\left\{z_{1}, \ldots, z_{2 n}\right\}$.

More precisely, if we denote by $\tilde{Z}_{n}$ the partition function, and

$$
Z_{n}=(-1)^{n(n-1) / 2}\left(q^{-1}-q\right)^{-n} \prod_{i=1}^{2 n} z_{i}^{-1 / 2} \tilde{Z}_{n}
$$

then $Z_{n}$ was identified at $q=e^{2 \pi i / 3}$ with the Schur function corresponding to the Young diagram $Y_{n}$ with two rows of length $n-1$, two rows of length $n-2, \ldots$, two rows of length 2 and two rows of length 1:

$$
Z_{n}\left(z_{1}, \ldots z_{2 n}\right)=s_{Y_{n}}\left(z_{1}, \ldots, z_{2 n}\right)=\frac{\operatorname{det}\left[z_{i}^{2 n-j+d_{j}}\right]}{\operatorname{det}\left[z_{i}^{2 n-j}\right]}
$$

where $d_{j}$ is the sequence $\{n-1, n-1, n-2, n-2, \ldots, 2,2,1,1,0,0\}$. This formula is proved in appendix $B$, though its explicit form will not be needed in what follows.

With this method we recover the unweighted enumeration by setting all $z_{i}=1$ :

$$
3^{-n(n-1) / 2} Z_{n}(1, \ldots, 1)=A_{n}
$$

where we recall that $A_{n}$ is the number of ASMs of size $n$ (as explained in 2.1).

The case of interest to us is when all $z_{i}=1$ except $z_{1}$ and $z_{2 n}$ :

$$
\begin{aligned}
z_{1} & =\frac{1+q t}{q+t} \\
z_{2 n} & =\frac{1+q u}{q+u}
\end{aligned}
$$

The EleCtronic Journal of COMBinatorics 15 (2008), \#R81 
Using the fact that $Z_{n}\left(z_{1}, \ldots, z_{2 n}\right)$ is a symmetric function of its arguments (see appendix B), we have

$$
\begin{aligned}
Z_{n}\left(z_{1}=\frac{1+q t}{q+t}, 1, \ldots, 1, z_{2 n}=\right. & \left.\frac{1+q u}{q+u}\right) \\
& =Z_{n}\left(z_{1}=\frac{1+q t}{q+t}, 1, \ldots, 1, z_{n}=\frac{1+q u}{q+u}, 1, \ldots, 1\right)
\end{aligned}
$$

The corresponding weights take the form

$$
\begin{aligned}
& a_{x}=q^{-\frac{1}{2}}-q^{\frac{1}{2}}\left(\frac{1+q x}{q+x}\right)=\frac{q^{\frac{1}{2}} x}{q+x}\left(q^{-1}-q\right) \\
& b_{x}=q^{-\frac{1}{2}}\left(\frac{1+q x}{q+x}\right)-q^{\frac{1}{2}}=\frac{q^{\frac{1}{2}}}{q+x}\left(q^{-1}-q\right) \\
& c_{x}=\left(q^{-1}-q\right) \sqrt{\frac{1+q x}{q+x}}
\end{aligned}
$$

The partition function $\tilde{Z}_{n}$ becomes

$$
\begin{aligned}
\tilde{Z}_{n} & =(-i \sqrt{3})^{n^{2}-2 n} \sum_{j, k} a_{t}^{j-1} b_{t}^{n-j} c_{t} a_{u}^{k-1} b_{u}^{n-k} c_{u} A_{n, j, k} \\
& =(-i \sqrt{3})^{n^{2}} \sqrt{\frac{1+q t}{q+t}} \sqrt{\frac{1+q u}{q+u}}\left(\frac{1}{q+t}\right)^{n-1}\left(\frac{1}{q+u}\right)^{n-1} q^{n-1} \sum_{j, k} t^{j-1} u^{k-1} A_{n, j, k}
\end{aligned}
$$

where $A_{n, j, k}$ is the number of ASMs of size $n$ such that the only 1 in the first row is in column $j$ and the only 1 in the last row is in column $n-k+1$.

The normalization factor is equal to: $(-1)^{n(n-1) / 2}(-i \sqrt{3})^{n} \sqrt{\frac{1+q t}{q+t}} \sqrt{\frac{1+q u}{q+u}}$, so we can finally compute

$$
\frac{\left(q^{2}(q+t)(q+u)\right)^{n-1}}{3^{n(n-1) / 2}} Z_{n}=\sum_{j, k} t^{j-1} u^{k-1} A_{n, j, k}=A_{n}(t, u)
$$

Note that if one uses instead $z_{2 n}=\frac{q+u}{1+q u}$, one gets the same formula, but with one index reversed

$$
\frac{\left(q^{2}(q+t)(1+q u)\right)^{n-1}}{3^{n(n-1) / 2}} Z_{n}=\tilde{A}_{n}(t, u)
$$




\subsection{Integral formula for refined ASM counting}

The traditional expression for the partition function of the $6-\mathrm{V}$ model is the already mentioned IK formula. We shall not use it here. We shall only need the following facts (true at $q=e^{2 \pi i / 3}$ ):

- $Z_{1}=1$.

- $Z_{n}\left(z_{1}, \ldots, z_{2 n}\right)$ is a polynomial of degree $n-1$ in each variable.

- The $Z_{n}$ satisfy the recursion relation for all $i, j=1, \ldots, 2 n$

$$
Z_{n}\left(z_{1}, \ldots, z_{j}=q^{2} z_{i}, \ldots, z_{2 n}\right)=\prod_{k \neq i, j}\left(q z_{i}-z_{k}\right) Z_{n-1}\left(z_{1}, \ldots, \hat{z}_{i}, \ldots, \hat{z}_{j}, \ldots, z_{2 n}\right)
$$

We recall how to prove them in appendix B for the sake of completeness.

Furthermore, we need the following lemma

Lemma 1. A polynomial $P$ of degree $n-1$ in each variable $z_{1}, \ldots, z_{2 n}$ which satisfies the "wheel condition"

$$
P\left(\ldots, z_{i}=z, \ldots, z_{j}=q^{2} z, \ldots, z_{k}=q^{4} z, \ldots\right)=0 \quad \text { for all } i<j<k
$$

is entirely determined by its $c_{n}:=(2 n) ! / n ! /(n+1)$ ! values at the following specializations: $\left(q^{\epsilon_{1}}, \ldots, q^{\epsilon_{2 n}}\right)$ for all possible choices of $\left\{\epsilon_{i}= \pm 1\right\}$ such that $\sum_{i=1}^{2 n} \epsilon_{i}=0$ and $\sum_{i=1}^{j} \epsilon_{i} \leq 0$ for all $j \leq 2 n$.

This lemma is proved in appendix C.

The strategy is now to introduce a certain integral representation of the partition function of the $6-\mathrm{V}$ model with DWBC, say $Z_{n}^{\prime}$

$$
\begin{aligned}
Z_{n}^{\prime}:=(-1)^{\left(\begin{array}{c}
n \\
2
\end{array}\right)} \prod_{i<j}^{2 n}\left(q z_{i}-q^{-1} z_{j}\right) \\
\oint \ldots \oint \prod_{l}^{n} \frac{d w_{l}}{2 \pi i} \frac{\left(q z_{2 l-1}-q^{-1} w_{l}\right) \prod_{l<m}\left(w_{m}-w_{l}\right)\left(q w_{l}-q^{-1} w_{m}\right)}{\prod_{i \leq 2 l-1}\left(w_{l}-z_{i}\right) \prod_{i \geq 2 l-1}\left(q w_{l}-q^{-1} z_{i}\right)}
\end{aligned}
$$

where the integration contours surround counterclockwise the $z_{i}$ (but not the $q^{-2} z_{i}$ ), and to show that $Z_{n}$ and $Z_{n}^{\prime}$ are both polynomials of degree $n-1$ in each variable which satisfy the "wheel condition" and coincide at the $c_{n}$ specializations of lemma 1. 
Let us first check that $Z_{n}$ satisfies the wheel condition. This is a direct consequence of Eq. (4.5) in which one sets $z_{k}=q^{4} z_{i}$. It is equally straightforward to calculate $Z_{n}$ at the $c_{n}$ points of the lemma. The computation goes inductively using Eq. (4.5) and it is left to the reader to check that

$$
Z_{n}\left(q^{\epsilon_{1}}, \ldots, q^{\epsilon_{2 n}}\right)=3^{\left(\begin{array}{c}
n \\
2
\end{array}\right)}
$$

We now show that $Z_{n}^{\prime}$ also satisfies the hypotheses of the lemma. We proceed in steps.

\section{$Z_{n}^{\prime}$ is a polynomial of degree $n-1$ in each variable.}

By applying the residue formula to Eq. (4.6) we obtain

$$
\begin{aligned}
& Z_{n}^{\prime}=(-1)^{\left(\begin{array}{c}
n \\
2
\end{array}\right)} \sum_{\substack{K=\left(k_{1}, \ldots, k_{n}\right) \\
k_{l} \neq k_{m} \text { if } l \neq m \\
k_{l} \leq 2 l-1}}(-1)^{s(K)} \prod_{l<m}\left(q z_{k_{l}}-q^{-1} z_{k_{m}}\right) \\
& \prod_{\substack{i<j \\
l \notin K \text { or }\left(i=k_{l} \text { and } j<2 l-1\right)}}\left(q z_{i}-q^{-1} z_{j}\right) \prod_{2 i-1 \neq k_{i}}\left(q z_{2 i-1}-q^{-1} z_{k_{i}}\right) \\
& \prod_{\substack{i \leq 2 j-1 \\
i \notin K \text { or } i>k_{j}}}\left(z_{k_{j}}-z_{i}\right)
\end{aligned}
$$

where $(-1)^{s(K)}$ is the sign of the permutation that orders the $k_{i}$. It is enough to prove that $\lim _{z_{k_{j}} \rightarrow z_{i}} Z_{n}^{\prime}$ exists; the verification is a tedious but easy calculation (see [6] for a similar check).

We can now consider the leading term in each variable $z_{i}$ in the summation of Eq. (4.7), depending on whether $i \in K$ or not; in both cases we find a degree $n-1$.

\section{$Z_{n}^{\prime}$ satisfies the wheel condition.}

Using the formula (4.7), we can verify that $Z_{n}^{\prime}$ is zero at $z_{k}=q^{2} z_{j}=q^{4} z_{i}$ for all $k>j>i$ : In fact, the term $\prod_{s<r \text { and } s \notin K}\left(q z_{s}-q^{-1} z_{r}\right)$ implies that $i$ and $j \in K$. As a consequence of the term $\prod_{l<m}\left(q z_{k_{l}}-q^{-1} z_{k_{m}}\right)$, we must have $i=k_{m}$ and $j=k_{l}$ with $l<m$, but, in this case, $j \leq 2 l-1<2 m-1$ proving that $Z_{n}^{\prime}$ satisfies the "wheel condition". 


\section{Recursion relation.}

We show that $Z_{n}^{\prime}$, at $q=e^{2 \pi i / 3}$, satisfies a weaker form of recursion relation (4.5). Let $j$ be an integer between 1 and $2 n-1$ and evaluate $Z_{n}^{\prime}$ at $z_{j+1}=q^{2} z_{j}$. We will perform the calculation for $j$ even.

If we look at formula (4.7) it is straightforward that all terms are zero except for $j=k_{m}$ and $j+1 \geq 2 m-1$, i.e. $j=k_{m}=2 m-2$. Using the fact that $z_{j+1}=q^{2} z_{j}$, we can derive

$$
\begin{aligned}
Z_{n \mid z_{j+1}=q^{2} z_{j}}^{\prime}= & \prod_{i<j}\left(q z_{i}-q^{-1} z_{j}\right)\left(q z_{i}-q z_{j}\right) \prod_{k>j+1}\left(q z_{j}-q^{-1} z_{k}\right)\left(z_{j}-q^{-1} z_{k}\right)(-1)^{\left(\begin{array}{c}
n \\
2
\end{array}\right)} \\
& \times \prod_{i<k \neq j, j+1}\left(q z_{i}-q^{-1} z_{k}\right) \oint \ldots \oint \prod_{l} \frac{d w_{l}}{2 \pi i} \prod_{l \neq m}\left(q z_{2 l-1}-q^{-1} w_{l}\right) \\
& \times \frac{\prod_{l<p \neq m}\left(w_{p}-w_{l}\right)\left(q w_{l}-q^{-1} w_{p}\right)\left(z_{j}-q^{-1} z_{j}\right)}{\prod_{l \neq m} \prod_{\substack{i \leq 2 l-1 \\
i \neq j, j+1}}\left(w_{l}-z_{i}\right) \prod_{\substack{i \geq 2 l-1 \\
i \neq j, j+1}}\left(q w_{l}-q^{-1} z_{i}\right)} \\
& \times \prod_{n>m} \frac{\left(w_{n}-z_{j}\right)\left(q z_{j}-q^{-1} w_{n}\right)}{\left(w_{n}-z_{j}\right)\left(w_{n}-q^{2} z_{j}\right)} \prod_{l<m} \frac{\left(z_{j}-w_{l}\right)\left(q w_{l}-q^{-1} z_{j}\right)}{\left(q w_{l}-q^{-1} z_{j}\right)\left(q w_{l}-q z_{j}\right)} \\
& \times \frac{1}{\left(z_{j}-q^{2} z_{j}\right) \prod_{i<j}\left(z_{j}-z_{i}\right) \prod_{k>j+1}\left(q z_{j}-q^{-1} z_{k}\right)}
\end{aligned}
$$

After multiple cancellations we get:

$$
Z_{n}^{\prime}\left(\ldots, z_{j}, z_{j+1}=q^{2} z_{j}, \ldots\right)=\prod_{i \neq j, j+1}\left(q z_{j}-z_{i}\right) Z_{n-1}^{\prime}\left(z_{1}, \ldots, z_{j-1}, z_{j+2}, \ldots, z_{2 n}\right)
$$

The formula actually holds for both parities of $j$; the proof for $j$ odd is similar.

\section{Calculating $Z_{n}^{\prime}$ at the $c_{n}$ points.}

Using the formula above, we can easily calculate $Z_{n}^{\prime}$ at the $c_{n}$ points of the lemma. One can always choose two consecutive variables which are $\left(q^{-1}, q\right)$ and apply the recursion relation above:

$$
\begin{aligned}
Z_{n}^{\prime}\left(\ldots, z_{j}=q^{-1}, z_{j+1}=q^{2} z_{j}=q, \ldots\right) & =\prod_{i \neq j, j+1}\left(1-z_{i}\right) Z_{n-1}^{\prime} \\
& =(1-q)^{n-1}\left(1-q^{-1}\right)^{n-1} Z_{n-1}^{\prime}
\end{aligned}
$$

The second equality uses the fact that there is the same number of $\epsilon_{i}=1$ and $\epsilon_{i}=-1$. Since we have $Z_{1}^{\prime}=1$, we obtain:

$$
Z_{n}^{\prime}=3^{\left(\begin{array}{c}
n \\
2
\end{array}\right)}
$$


We finally conclude, by applying lemma 1 , that

$$
Z_{n}=Z_{n}^{\prime}
$$

Starting from our new integral formula for the partition function of the 6-Vertex model (4.6), we are now in a position to calculate

$$
\frac{\left(q^{2}(q+x)(1+q y)\right)^{n-1}}{\left(q-q^{-1}\right)^{n(n-1)}} Z_{n}\left(\frac{1+q x}{q+x}, 1, \ldots, 1, \frac{q+y}{1+q y}\right)
$$

After some tedious computations and using new variables

$$
u_{i}=\frac{w_{i}-1}{q w_{i}-q^{-1}}
$$

we obtain:

$$
(y+x-y x) \oint \ldots \oint \prod_{l}^{n} \frac{d u_{l}}{2 \pi i} \frac{1}{u_{l}^{2 l-2}} \frac{\prod_{l<m}\left(u_{m}-u_{l}\right)\left(1+u_{m}+u_{m} u_{l}\right)}{\left(1+u_{l}-x\right)\left(1+u_{l}(1-y)\right)} \prod_{j=2}^{n}\left(1+u_{j}\right)
$$

where the integral contours surround counterclockwise $u_{i}=0$ and $u_{i}=x-1$ (and not $1 /(y-1))$.

To simplify our calculation we integrate on $u_{1}$ :

$$
\tilde{A}_{n}(x, y)=\oint \ldots \oint \prod_{l=2}^{n} \frac{d u_{l}}{2 \pi i} \frac{\left(1+u_{l}\right)\left(1+x u_{l}\right)}{u_{l}^{2 l-2}\left(1+u_{l}(1-y)\right)} \prod_{l<m}^{n}\left(u_{m}-u_{l}\right)\left(1+u_{m}+u_{m} u_{l}\right)
$$

where the contours surround the remaining poles at $u_{i}=0$ only.

\subsection{Integral formula for refined NILP counting}

We will derive a contour integral formula for the generating polynomial $N_{10}^{\prime}\left(t_{0}, t_{1}, \ldots\right.$, $t_{n-1}$ ) of our NILPs with a weight $t_{i}$ per vertical step in the $i^{\text {th }}$ slice (between $y=1-i$ and $y=-i$ ). We use the Lindström-Gessel-Viennot formula [13, 7] (see also the third chapter of [2]):

$$
N_{10}^{\prime}\left(t_{0}, t_{1}, \ldots, t_{n-1}\right)=\sum_{\substack{1=r_{1}<\ldots<r_{n-1} \\ r_{i} \leq 2 i+1 \\ r_{i+1}-r_{i} \text { odd }}} \operatorname{det}\left[\mathcal{P}_{i, r_{j}}\right]
$$


where $\mathcal{P}_{i, r}$ is the weighted sum over all possible lattice paths from $(i,-i)$ to $(r+1,1)$. Such paths counts with $r-i+1$ diagonal steps and $2 i-r$ vertical ones, hence:

$$
\mathcal{P}_{i, r}=\sum_{0 \leq i_{1}<\ldots<i_{2 i-r} \leq i} \prod_{l=1}^{2 i-r} t_{i_{l}}=\left.\prod_{k=0}^{i}\left(1+t_{k} u\right)\right|_{u^{2 i-r}}
$$

where the subscript $u^{2 i-r}$ stands for the coefficient of the corresponding power of $u$ in the polynomial.

We can reintroduce the path beginning at $(0,0)$ and rewrite the equation as a contour integral:

$$
N_{10}^{\prime}\left(t_{0}, t_{1}, \ldots, t_{n-1}\right)=\oint \ldots \oint \prod_{i=1}^{n} \frac{d u_{i}}{2 \pi i u_{i}^{2 i-1}} \prod_{k=0}^{i-1}\left(1+t_{k} u_{i}\right) \sum_{\substack{0=r_{0}<r_{1}<\ldots<r_{n-1} \\ r_{i+1}-r_{i} \text { odd }}} \operatorname{det}\left[u_{i}^{r_{j-1}}\right]
$$

where the paths of integrations are small counterclockwise circles around zero.

The last sum can be evaluated as a standard result for the sum over all Schur functions corresponding to even partitions (see exercise 4.3.9 in [2]):

$$
\sum_{\substack{0=r_{0}<r_{1}<\ldots<r_{n-1} \\ r_{i+1}-r_{i} \text { odd }}} \operatorname{det}\left[u_{i}^{r_{j-1}}\right]=\frac{\prod_{j>i}\left(u_{j}-u_{i}\right)}{\prod_{j \geq i}\left(1-u_{j} u_{i}\right)}
$$

where we have relaxed the condition $r_{0}=0$ into $r_{0} \geq 0$ and even, since this does not affect the integral.

The integral can thus be transformed as follows:

$$
N_{10}^{\prime}\left(t_{0}, t_{1}, \ldots, t_{n-1}\right)=\oint \ldots \oint \prod_{i=1}^{n} \frac{d u_{i}}{2 \pi i u_{i}^{2 i-1}} \frac{1}{1-u_{i}^{2}} \prod_{k=0}^{i-1}\left(1+t_{k} u_{i}\right) \prod_{j>i} \frac{u_{j}-u_{i}}{1-u_{j} u_{i}}
$$

We are mainly interested in the case where $t_{0}=t, t_{1}=s$ and all the others $t_{i}$ equal 1. In this case, we rewrite the equation:

$$
\begin{aligned}
& N_{10}^{\prime}(t, s, 1, \ldots, 1):=U_{n}^{0,1}(t, s) \\
& \quad=\oint \ldots \oint \prod_{i=1}^{n} \frac{d u_{i}}{2 \pi i u_{i}^{2 i-1}} \frac{1}{1-u_{i}^{2}}\left(1+t u_{i}\right)\left(1+s u_{i}\right)_{\hat{1}}\left(1+u_{i}\right)_{\hat{1}}^{i-2} \prod_{j>i} \frac{u_{j}-u_{i}}{1-u_{j} u_{i}}
\end{aligned}
$$

where $\hat{1}$ means that we exclude the term corresponding to $u_{1}$. 


\subsection{Equality of integral formulae}

At this point, we have two integral expressions, $A_{n}(x, y)$ (in equation (4.9)) and $U_{n}^{0,1}(x, y)$ (in equation (4.14)) and we want to prove that they are the same. The first step is to integrate over $u_{1}$ the expression (4.14):

$$
U_{n}^{0,1}(x, y)=\oint \ldots \oint \prod_{i=2}^{n} \frac{d u_{i}}{2 \pi i u_{i}^{2 i-2}}\left(1+x u_{i}\right)\left(1+y u_{i}\right)\left(1+u_{i}\right)^{i-2} \frac{\prod_{i<j}\left(u_{j}-u_{i}\right)}{\prod_{i \leq j}\left(1-u_{j} u_{i}\right)}
$$

At this stage we use the following identity:

$$
\begin{aligned}
\oint \ldots \oint \prod_{i} \frac{d u_{i}}{2 \pi i} \frac{\varphi(u)}{u_{i}^{2 i}} \prod_{i<j}\left(u_{j}-u_{i}\right)\left(1+\tau u_{j}+u_{i} u_{j}\right) \\
\quad=\oint \ldots \oint \prod_{i} \frac{d u_{i}}{2 \pi i} \varphi(u) \frac{\left(1+\tau u_{i}\right)^{i-1}}{u_{i}^{2 i}} \frac{\prod_{i<j}\left(u_{j}-u_{i}\right)}{\prod_{i \leq j}\left(1-u_{i} u_{j}\right)}
\end{aligned}
$$

for any $\varphi(u)$ completely symmetric in $\left(u_{1}, u_{2}, \ldots, u_{n}\right)$ and without poles in a neighborhood of zero. This was conjectured in [6] and proved in [24]. We present in appendix D an independent proof of a stronger formula that implies Eq. (4.16).

If we shift the indexes $(i-1) \rightarrow i$, consider $\tau=1$ and set $\varphi(u)=\prod_{i=1}^{n-1}(1+$ $\left.x u_{i}\right)\left(1+y u_{i}\right)$ we can apply the equality:

$$
U_{n}^{0,1}(x, y)=\oint \ldots \oint \prod_{i=2}^{n} \frac{d u_{i}}{2 \pi i u_{i}^{2 i-2}}\left(1+x u_{i}\right)\left(1+y u_{i}\right) \prod_{i<j}\left(u_{j}-u_{i}\right)\left(1+u_{j}+u_{j} u_{i}\right)
$$

Notice that the two integrals are the same, except for the pieces $\frac{\left(1+u_{l}\right)}{\left(1+u_{l}(1-y)\right)}$ versus $1+y u_{l}$. Unsurprisingly, we find that is possible to write both integrals as special cases of the same integral:

$$
I_{n}(x, y)=\oint \ldots \oint \prod_{l=1}^{n-1} \frac{d u_{l}}{2 \pi i} \frac{\left(1+u_{l}+a_{l} u_{l}^{2}\right)\left(1+x u_{l}\right)}{u_{l}^{2 l}\left(1+u_{l}(1-y)\right)} \prod_{l<m}^{n-1}\left(u_{m}-u_{l}\right)\left(1+u_{m}+u_{m} u_{l}\right)
$$

which takes the value of $\tilde{A}_{n}(x, y)$ if $a_{l}=0$ for all $l$ and takes the value of $U^{0,1}(x, y)$ if $a_{l}=y(1-y)$ for all $l$.

More surprising is the fact that $I_{n}$ does not depend on the $a_{i}$. We shall show by induction on $i$ that $I_{n}$ is independent of $a_{i}$, noting that it is a polynomial in $a_{i}$ of degree at most 1 . 
Let us first differentiate $I_{n}$ with respect to $a_{1}$ :

$$
\begin{aligned}
\frac{d}{d a_{1}} I_{n}(x, y) & =\oint \frac{d u_{1}}{2 \pi i} \frac{\left(1+x u_{1}\right)}{\left(1+u_{1}(1-y)\right)} \\
& \times \oint \ldots \oint \prod_{l=2}^{n-1} \frac{d u_{l}}{2 \pi i} \frac{\left(1+u_{l}+a_{l} u_{l}^{2}\right)\left(1+x u_{l}\right)}{u_{l}^{2 l}\left(1+u_{l}(1-y)\right)} \prod_{m<l}^{n-1}\left(u_{l}-u_{m}\right)\left(1+u_{l}+u_{m} u_{l}\right)
\end{aligned}
$$

but, this integral has no poles at $u_{1}$ so it vanishes.

Let us now assume by induction hypothesis that $I_{n}$ does not depend on the first $(i-1) a_{j}$, and prove that the expression (4.18) does not depend on $a_{i}$ either. As the integral does not depend on $a_{j}$ for all $j<i$ we can set all $a_{j}=0$ (for $j<i$ ).

If we differentiate now with respect to $a_{i}$ and look at what happens in the integration up to $u_{i}$. We find an expression of the type:

$$
J_{i}=\oint \frac{d u_{i}}{2 \pi i u_{i}^{2 i-2}} \oint \cdots \oint \prod_{j=1}^{i-1} \frac{d u_{j}}{2 \pi i} \frac{1+u_{j}}{u_{j}^{2 j}} \Theta_{i} A_{i}
$$

where $A_{i}$ is some anti-symmetric function in the $u_{j}$ for all $j \leq i$ without any poles in the integration domain, and $\Theta_{i}=\prod_{j<i}\left(1+u_{i}+u_{j} u_{i}\right)$.

To prove that this integral is always zero we shall proceed once again by induction. The first one, $J_{1}$, is zero because it has no poles:

$$
J_{1}=\oint \frac{d u_{1}}{2 \pi i} A_{1}\left(x_{1}\right)=0
$$

Let $J_{i-1}=0$. All the poles are at 0 , the $A_{i}$ is anti-symmetric between $u_{i}$ and $u_{i-1}$, so we can take advantage of the fact that the $u_{i}$ appears with the same degree as $u_{i-1}$ in the denominator to erase all the symmetric terms in the expression $(1+$ $\left.u_{i-1}\right)\left(1+u_{i}+u_{i-1} u_{1}\right)$ and get $u_{i} u_{i-1}^{2}$ :

$$
J_{i}=\oint \frac{d u_{i}}{2 \pi i u_{i}^{2 i-3}} \oint \frac{d u_{i-1}}{2 \pi i u_{i-1}^{2 i-4}} \oint \ldots \oint \prod_{j=1}^{i-2} \frac{d u_{j}}{2 \pi i} \frac{1+u_{j}}{u_{j}^{2 j}} \hat{\Theta}_{i} A_{i}
$$

where the hat in $\hat{\Theta}_{i}$ means that the term $\left(1+u_{i}+u_{i-1} u_{i}\right)$ is skipped ${ }^{1}$. The integral does not have yet the desired form, i.e. $J_{i-1}$, it is missing the term $\left(1+u_{i}+u_{i-1} u_{i}\right)$,

\footnotetext{
${ }^{1}$ Note that $\hat{\Theta}_{i}$ is symmetric between $u_{i-1}$ and $u_{i}$.
} 
so we add and subtract it:

$$
\begin{aligned}
J_{i}= & \oint \ldots \oint \frac{d u_{i}}{2 \pi i u_{i}^{2 i-3}} \frac{d u_{i-1}}{2 \pi i u_{i-1}^{2 i-4}} \prod_{j=1}^{i-2} \frac{d u_{j}}{2 \pi i} \frac{1+u_{j}}{u_{j}^{2 j}}\left(1+u_{i}+u_{i} u_{i-1}-u_{i}-u_{i} u_{i-1}\right) \hat{\Theta}_{i} A_{i} \\
= & \oint \ldots \oint \frac{d u_{i}}{2 \pi i u_{i}^{2 i-3}} \frac{d u_{i-1}}{2 \pi i u_{i-1}^{2 i-4}} \prod_{j=1}^{i-2} \frac{d u_{j}}{2 \pi i} \frac{1+u_{j}}{u_{j}^{2 j}} \Theta_{i} A_{i} \\
& -\oint \ldots \oint \frac{d u_{i}}{2 \pi i u_{i}^{2 i-4}} \frac{d u_{i-1}}{2 \pi i u_{i-1}^{2 i-4}} \prod_{j=1}^{i-2} \frac{d u_{j}}{2 \pi i} \frac{1+u_{j}}{u_{j}^{2 j}}\left(1+u_{i-1}\right) \hat{\Theta}_{i} A_{i}
\end{aligned}
$$

The first term is already in the form of $J_{i-1}$. The second term is almost symmetric between $u_{i}$ and $u_{i-1}$, using the same method as in (4.21) we can transform $-\left(1+u_{i-1}\right)$ to $1+u_{i}+u_{i} u_{i-1}$; in this way, we recover the symmetry needed so that we can write $J_{i}$ as an integral in $u_{i}$ of some function multiplied by $J_{i-1}$, which is zero. As a consequence $J_{i}$ is also zero for all $i$, i.e. $I_{n}(x, y)$ does not depend on any $a_{i}$. We conclude that

$$
\tilde{A}_{n}(x, y)=U_{n}^{0,1}(x, y)
$$

\section{A Formulating the conjecture directly in terms of TSSCPPs}

We have used the NILP formulation throughout this paper (in particular, to prove the main theorem), whereas Mills, Rumsey and Robbins use the language of TSSCPPs. In A.1 we first describe the theorem in a more general form, and then prove that we can reduce it to the one presented in 3.3. We then reformulate in A.2 our theorem in the language of $[16,20]$.

\section{A.1 Extending the theorem}

Let $A_{n}(x, y)$ and $\tilde{A}_{n}(x, y)$ be the same as defined in 3.1. We use the same NILPs with the extra-step as in 3.2 .

We now introduce a function $u_{n}^{k}(\alpha)$, where $\alpha$ is a NILP, which counts the number of vertical steps in the extra-step if $k=0$; otherwise it counts the number of vertical steps in the $\max \{1, t-k+1\}$-th step of the path starting at $(t,-t)$, as shown on figure 8 . 


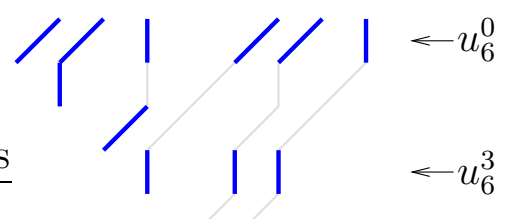

Figure 8: Let $\alpha$ be the NILP represented here. In order to calculate $u_{6}^{0}(\alpha)$ and $u_{6}^{3}(\alpha)$ we highlight the extra-steps and the $\max \{1, t-3+1\}$-th step of the path starting at $(t,-t)$. Here we have $u_{6}^{0}(\alpha)=2$ and $u_{6}^{3}(\alpha)=4$.

We can next define the function $U_{n}^{i}(x)$ :

$$
U_{n}^{i}(x):=\sum_{k} U_{n, k}^{i} x^{k}:=\sum_{\alpha} x^{u_{n}^{i}(\alpha)}
$$

and more complex functions $U_{n}^{i, j}(x, y)$ :

$$
U_{n}^{i, j}(x, y):=\sum_{k} U_{n, k, l}^{i, j} x^{k} y^{l}:=\sum_{\alpha} x^{u_{n}^{i}(\alpha)} y^{u_{n}^{j}(\alpha)}
$$

We could generalize these even more, introducing more indices, but this is general enough for our purposes. With these new functions we can rewrite our theorem:

\section{Theorem.}

$$
\begin{aligned}
& \tilde{A}_{n}(x, y)=U_{n}^{0, j}(x, y) \\
& A_{n}(x, y)=U_{n}^{1, i}(x, y)
\end{aligned}
$$

where $j=1,2 \ldots$ and $i=2,3 \ldots$. If we choose $U_{n}^{0,1}$ we have the theorem as stated before.

On order to reduce this to our previous result, it is enough to prove that $U_{n}^{0, i}$ does not depend in $i$ and that $U_{n, k, j}^{0, i}=U_{n, n-k-1, j}^{1, i}($ for $i \geq 2)$.

\section{$i$ independence of $U_{n}^{0, i}$.}

For the first equality we introduce a function $g$ as explained on figure 9. This function interchanges the number of vertical steps in two consecutive rows leaving invariant all the other rows. This function has the important property $g \circ g=I d$. So, it is straightforward from this that $U_{n}^{0, i}=U_{n}^{0, i+1}$, with $i$ greater than 0 . 


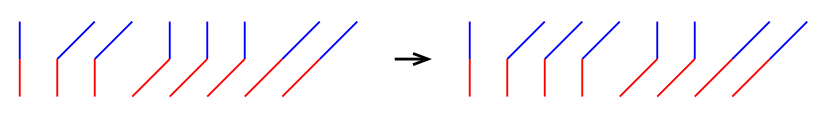

Figure 9: We can group the double steps in islands, such that all the starting points (of the double steps) are consecutive. These doubles steps are, necessarily, ordered in $r$ double vertical steps, $s$ vertical-diagonal steps, $t$ diagonal-vertical steps and $u$ double diagonal steps. Our function $g$ interchanges $s$ with $t$ at each island, so that we interchange the number of vertical steps between the two rows.
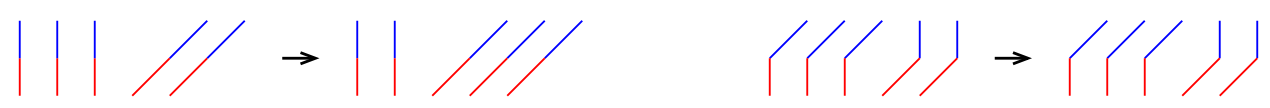

Figure 10: In order to satisfy the extra-step rules we can only build two type of islands, one made of $r$ double vertical steps and $s$ double diagonal steps, and the other type made of $t$ vertical-diagonal steps and $u$ diagonal-vertical steps. Our function $h$ interchange simply $r$ with $s$. It is important to note that the first path is always invariant under $h$ (it is always of the type vertical-diagonal or the inverse).

$U_{n, k, j}^{0, i}=U_{n, n-k-1, j}^{1, i}$ for $i>1$.

The proof follows the same structure as the former. We construct again a function $h$ such that $h \circ h=I d$, which interchanges the number of vertical steps at the extrastep with the number of diagonal steps at the last step (before the extra-step). This function is obviously a bijection and it leaves invariant all the rows except the last one and the extra one because it is applied at the top of the diagrams as can be seen on figure 10. An important remark is that the first path is always invariant under $h$ because it is of the type vertical-diagonal or diagonal-vertical. This proves our equality.

In conclusion, all these variations ((A.3) and (A.4)) are truly the same, and we can concentrate on only one version.

\section{A.2 The conjecture in terms of TSSCPPs}

Mills, Robbins and Rumsey conjectured this theorem by means of TSSCPPs, not NILPs, but behind the different formulations lies the same result. To show that, we describe some of the content of [16] and explain the equivalence.

Recall that TSSCPPs can be represented as $2 n \times 2 n$ matrices $a$, as in Eq. (2.2). In [16] is introduced a quantity which we shall denote by $u_{n}^{k}(a)$, and which depends 


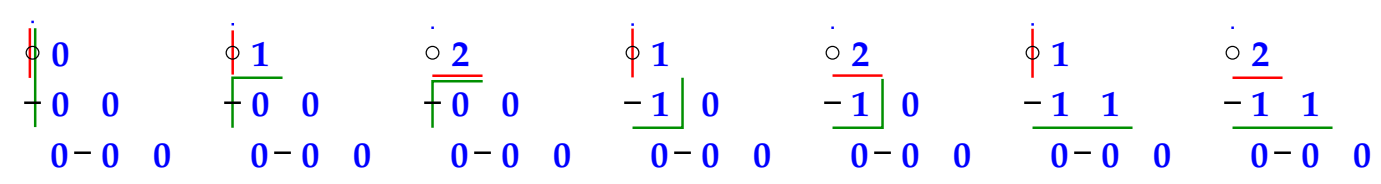

Figure 11: We can see on this figure what the function $u_{3}^{2}$ counts. The signs minus represents the part: $a_{t, t-k}-a_{t, t-k+1}$, so they count the vertical steps, and the little circles represents $\#\left\{a_{t, n+1} \mid a_{t, n+1}<2 n-t\right\}$. If we stretch our diagrams to obtain the NILPs we recover our definition of $u_{n}^{k}$.

on the upper-left $n \times n$ submatrix of $a$ :

$$
u_{n}^{k}(a)=\sum_{t=1}^{n-k+1}\left(a_{t, t+k-1}-a_{t, t+k}\right)+\sum_{t=n-k+2}^{n} \#\left\{a_{t, n} \mid a_{t, n}>2 n-t+1\right\}
$$

where \# means cardinality, and where conventionally, $a_{t, n+1}:=2 n-t+1$ in this equation. Also defined is the function:

$$
U_{n}^{i, \ldots k}(x, \ldots, z)=\sum_{a} x^{u_{n}^{i}(a)} \ldots z^{u_{n}^{k}(a)} \quad \text { for all } i, \ldots, k \in\{1, \ldots, n+1\}
$$

We claim that these are our functions $u$ and $U$ defined above. To make the connection, reexpress this function in terms of the lower-right $n \times n$ submatrix of $a$ :

$$
u_{n}^{k}(a)=\sum_{t=n+k}^{2 n}\left(a_{t, t-k}-a_{t, t-k+1}\right)+\sum_{t=n+1}^{n+k-1} \#\left\{a_{t, n+1} \mid a_{t, n+1}<2 n-t\right\}
$$

where we replace $a_{t, n}$ with $2 n-t$. What this function counts is described on figure 11 . Finally, if we shift the diagrams to obtain NILPs we recover our functions $U_{n}^{k}$ as expected.

As a final remark, in the article [20] three functions are defined: $f_{1}, f_{2}$ and $f_{3}$ and the conjecture is stated with any two of them. In fact, $f_{1}$ is connected with the $u_{n}^{0}, f_{2}$ with the $u_{n}^{1}$ and $f_{3}$ with $u_{n}^{n}$, as can be seen using the same procedure.

\section{B Properties of the 6-Vertex model partition function}

Consider, as in section 4.1, the 6-Vertex model with Domain Wall Boundary Conditions. Let $\tilde{Z}_{n}$ be its partition function (with Boltzmann weights given by Fig. 7), and $Z_{n}$ to be $\tilde{Z}_{n}$ divided by the normalization factor $(-1)^{n(n-1) / 2}\left(q^{-1}-q\right)^{n} \prod_{i=1}^{2 n} z_{i}^{1 / 2}$. 


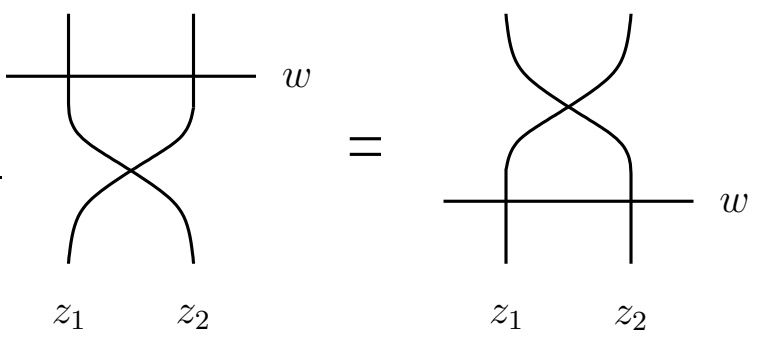

Figure 12: Yang-Baxter equation. Summation over arrows of the internal edges is implied, while the external arrows are fixed and the equality holds for any choice of them.

The model thus defined satisfies the following essential property (Yang-Baxter equation) shown on Fig. 12. The vertex with diagonal edges is assigned weights (the so-called $R$ matrix) which are those of Fig. 7 in which we have rotated the picture 45 degrees clockwise, and with parameters $z_{1}, q^{1 / 2} z_{2}$. parameter. In fact here we do not need the explicit expression of the $R$ matrix, only that it is invariant by reversal of all arrows and that it satisfies the ice rule i.e. there are as many outgoing arrows as incoming arrows. Since the Yang-Baxter equation is invariant by change of normalization of $R$, we can divide all weights by $b$ in such a way that $R_{\uparrow \uparrow}^{\uparrow \uparrow}=R_{\downarrow \downarrow}^{\downarrow \downarrow}=1$, with obvious notations.

\section{B.1 Korepin recursion relation}

In this paragraph, $q$ is kept arbitrary. We shall now list the following four properties which determine entirely $Z_{n}$ and only sketch their proof (since they have been reproved many times since their original appearance [11], see for example [12, 10])

- $Z_{1}=1$.

This is by definition.

- $Z_{n}$ is a symmetric function of the variables $\left\{z_{1}, \ldots, z_{n}\right\}$ and $\left\{z_{n+1}, \ldots, z_{2 n}\right\}$.

It is sufficient to prove that exchange of $z_{i}$ and $z_{i+1}$ (for $1 \leq i<n$ ) leaves the partition function unchanged. This can be obtained by repeated use of the Yang-Baxter property. Multiplying the partition function by $R\left(z_{i+1} / z_{i}\right)$ and 
noting that it is unchanged, we find

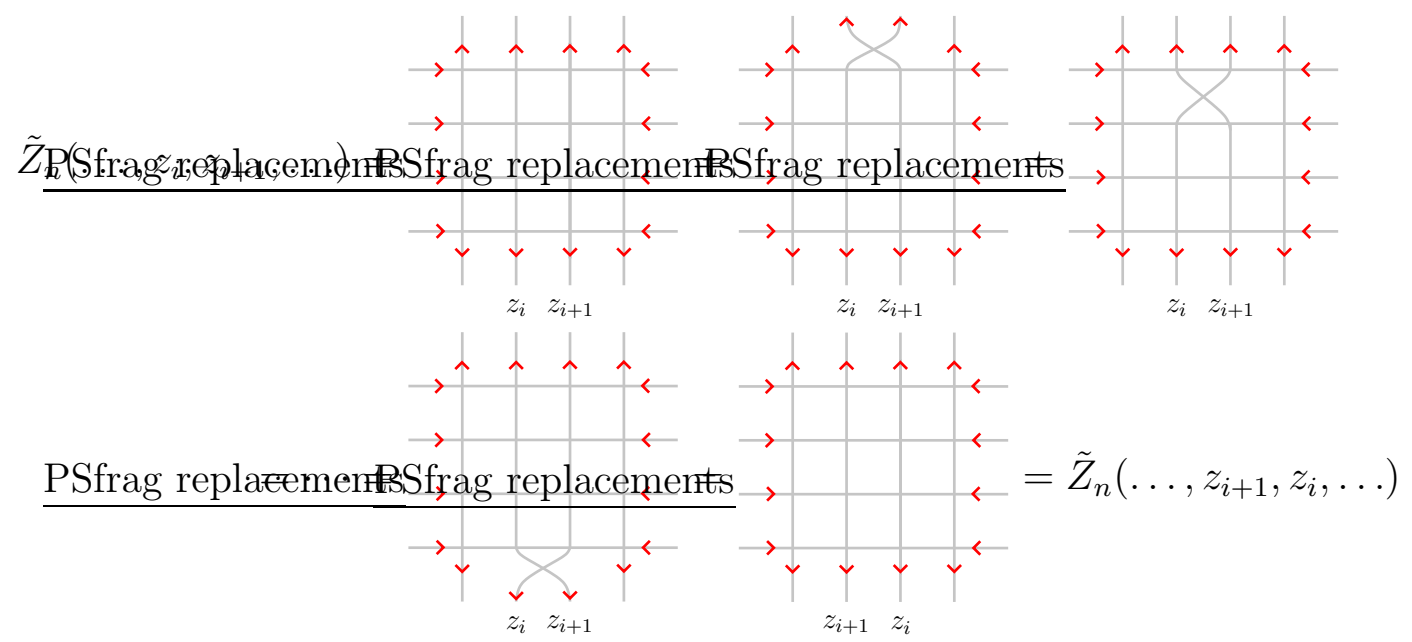

and similarly for the $\left\{z_{n+1}, \ldots, z_{2 n}\right\}$.

- $Z_{n}\left(z_{1}, \ldots, z_{2 n}\right)$ is a polynomial of degree (at most) $n-1$ in each variable.

Let us choose one configuration. Then the only weights which depend on $z_{i}$ are the $n$ weights on row $i$. Since the outgoing arrows are in opposite directions, the number of vertices of type $c$ on this row is odd, and in particular is at least 1. Power counting then shows that the contribution to the partition function of any configuration is of the form $z_{i}^{1 / 2}$ times a polynomial of $z_{i}$ of degree at most $n-1$. Summing over all configurations and removing $z_{i}^{1 / 2}$ by definition of $Z_{n}$, we obtain the desired property.

- The $Z_{n}$ obey the following recursion relation:

$$
\begin{aligned}
& Z_{n}\left(z_{1}, \ldots, z_{n} ; z_{n+1}=q^{-1} z_{1}, \ldots, z_{2 n}\right) \\
& =q^{-n+1} \prod_{j=2}^{n}\left(z_{1}-q^{2} z_{j}\right) \prod_{j=n+2}^{2 n}\left(z_{1}-q^{-1} z_{j}\right) Z_{n-1}\left(z_{2}, \ldots, z_{n} ; z_{n+2}, \ldots, z_{2 n}\right)
\end{aligned}
$$

Since $z_{n+1}=q^{-1} z_{1}$ implies $a\left(z_{n+1}, z_{1}\right)=0$, by inspection all configurations with non-zero weights are of the form shown on Fig. 13. This produces the following identity for unnormalized partition functions

$$
\begin{aligned}
& \tilde{Z}_{n}\left(z_{1}, \ldots, z_{n} ; z_{n+1}=q^{-1} z_{1}, \ldots, z_{2 n}\right)=\left(q^{-1}-q\right) z_{1} q^{-1 / 2} \\
& \times \prod_{j=2}^{n}\left(q^{-3 / 2} z_{1}-q^{1 / 2} z_{j}\right) \prod_{j=n+2}^{2 n}\left(q^{-1 / 2} z_{j}-q^{1 / 2} z_{1}\right) \tilde{Z}_{n-1}\left(z_{2}, \ldots, z_{n} ; z_{n+2}, \ldots, z_{2 n}\right)
\end{aligned}
$$




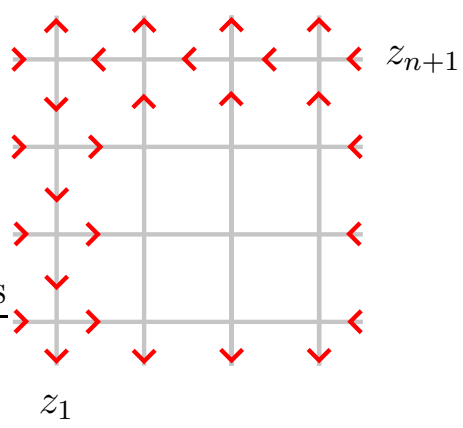

Figure 13: Graphical proof of the recursion relation.

which in turns leads to the recursion relation above for the $Z_{n}$.

Note that by the symmetry property, Eq. (B.1) fixes $Z_{n}$ at $n$ distinct values of $z_{n+1}=q^{-1} z_{i}, i=1, \ldots, n$. Since $Z_{n}$ is of degree $n-1$ in $z_{n+1}$, it is entirely determined by it.

\section{B.2 Cubic root of unity case}

Let us set $q=e^{2 \pi i / 3}$. First, once can simplify the recursion relation (B.1) to

$$
Z_{n}\left(z_{1}, \ldots, z_{n+1}=q^{2} z_{1}, \ldots, z_{2 n}\right)=\prod_{j \neq 1, n+1}\left(q^{-2} z_{1}-z_{j}\right) Z_{n-1}\left(z_{2}, \ldots, z_{n}, z_{n+2}, \ldots, z_{2 n}\right)
$$

Secondly, one wishes to show the enhanced symmetry property of $Z_{n}$ in the full set of variables $\left\{z_{1}, \ldots, z_{2 n}\right\}$. For this, it is simplest to prove Eq. (4.1), which displays explicitly this symmetry. Let us show that the Schur function $s_{Y_{n}}\left(z_{1}, \ldots, z_{2 n}\right)$ satisfies all the properties of the previous section.

$s_{Y_{0}}=1$ by definition. $s_{Y_{n}}$ is symmetric in all variables (which is what we want to prove for $Z_{n}$ ), and therefore in particular symmetric in the $\left\{z_{1}, \ldots, z_{n}\right\}$ and $\left\{z_{n+1}, \ldots, z_{2 n}\right\}$. It is a polynomial of degree $n-1$ in each variable because the width of the Young diagram $Y_{n}$ is $n-1$. Finally, to obtain the recursion relation, we note that as soon as $\left(z_{i}, z_{j}, z_{k}\right)=\left(z, q^{2} z, q^{4} z\right)$ for distinct $i, j, k$, the three corresponding rows in the numerator of Eq. (4.1) are linearly dependent so that the numerator vanishes while the denominator does not. Thus, at $z_{j}=q^{2} z_{i}, i \neq j$,

$$
s_{Y_{n}}\left(z_{1}, \ldots, z_{j}=q^{2} z_{i}, \ldots, z_{2 n}\right)=\prod_{k \neq i, j}\left(q^{-2} z_{i}-z_{k}\right) Z_{n-1}^{\prime \prime}\left(z_{2}, \ldots, \hat{z}_{i}, \ldots, \hat{z}_{j}, \ldots, z_{2 n}\right)
$$


where $Z_{n-1}^{\prime \prime}$ does not depend on $z_{i}$ because the $2 n-2$ prefactors exhaust the degree in $z_{i}$.

Now set $z_{i}=0$ : the Schur function has $2 n-2$ remaining arguments, so the full column of length $2 n-2$ can be factored out and we are left with the Young diagram $Y_{n-1}$ :

$$
s_{Y_{n}}\left(z_{1}, \ldots, z_{i}=0, \ldots, z_{j}=0, \ldots, z_{2 n}\right)=\prod_{k \neq i, j} z_{k} s_{Y_{n-1}}\left(z_{2}, \ldots, \hat{z}_{i}, \ldots, \hat{z}_{j}, \ldots, z_{2 n}\right)
$$

By comparison, we conclude that $Z_{n-1}^{\prime \prime}=s_{Y_{n-1}}$, so that $s_{Y_{n}}$ satisfies the desired recursion relation.

We conclude that $s_{Y_{n}}$ satisfies all the properties of the previous section, which determine uniquely $Z_{n}$. Thus, Eq. (4.1) holds.

\section{The space of polynomials satisfying the wheel condition}

In order to prove that $Z_{n}^{\prime}$ (defined in (4.6)) is the partition function of the 6 - $\mathrm{V}$ model, we need to prove lemma 1 . That is, a polynomial $P$ of degree (at most) $n-1$ in each variable $z_{1}, \ldots, z_{2 n}$ satisfying the "wheel condition" is entirely determined by its values at the following specializations: $\left(q^{\epsilon_{1}}, \ldots, q^{\epsilon_{2 n}}\right)$ for all possible choices of $\left\{\epsilon_{i}= \pm 1\right\}$ such that $\sum_{i=1}^{2 n} \epsilon_{i}=0$ and $\sum_{i=1}^{j} \epsilon_{i} \leq 0$ for all $j \leq 2 n$ (these are just increments of Dyck paths).

Or equivalently, if a polynomial satisfies these conditions and is zero at all the specializations, then it is identically zero. For example, at $n=1$ the polynomial is of degree 0 i.e. a constant, and as it vanishes at $\left(z_{1}, z_{2}\right)=\left(q^{-1}, q\right)$ it is identically zero.

We now proceed by induction. We suppose that the lemma is true for $n<p$. Let $\phi_{p}$ be a polynomial of degree $(p-1)$ at each variable which is zero at all specializations. The polynomial satisfies the "wheel condition" at $z_{i+1}=q^{2} z_{i}$, so we can write

$$
\phi_{p}\left(z_{1}, \ldots, z_{2 p}\right){ }_{\mid z_{i+1}=q^{2} z_{i}}=\prod_{j \neq i, i+1}\left(q z_{i}-z_{j}\right) \psi_{p-1}\left(z_{1}, \ldots, z_{i-1}, z_{i+2}, \ldots, z_{2 p}\right)
$$

where $\psi_{p-1}$ is a function of degree $p-2$ in each $z_{j}$ (except $z_{i}$ and $z_{i+1}$ ) which still follows the "wheel condition". Furthermore, let $\pi_{p}$ be a specialization which has $\left(z_{i}, z_{i+1}\right)=\left(q^{-1}, q\right)$ and $\pi_{p-1}^{\prime}$ the same specialization but without $z_{i}$ and $z_{i+1}$. We apply (C.1):

$$
\phi_{p}\left(\pi_{p}\right)=(1-q)^{n-1}\left(1-q^{-1}\right)^{n-1} \psi_{p-1}\left(\pi_{p-1}^{\prime}\right)=0
$$


The mapping $\pi_{p} \mapsto \pi_{p-1}$ is a bijection from Dyck paths with $\left(q^{-1}, q\right)$ at locations $(i, i+1)$ to all Dyck paths. Thus our induction hypothesis applies, and $\psi_{p-1}=0$.

Therefore, one can write:

$$
\phi_{p}=\prod_{i=1}^{2 n-1}\left(z_{i+1}-q^{2} z_{i}\right) \phi_{p}^{(1)}
$$

where $\phi_{p}^{(1)}$ is a polynomial of degree $\delta_{1}=\delta_{2 p}=p-2$ at $z_{1}$ and $z_{2 p}$ and $\delta_{i}=p-3$ at all the other variables which follows a weak version of the "wheel condition":

$$
\phi_{p \mid z_{k}=q^{2} z_{j}=q^{4} z_{i}}^{(1)}=0 \quad \text { for all } k \geq j+2 \geq i+4
$$

This implies:

$$
\phi_{p \mid z_{i+2}=q^{2} z_{i}}^{(1)}=\prod_{j \notin[i-1, i+3]}\left(q z_{1}-z_{j}\right) \psi_{p}^{(1, i)}
$$

By degree counting in $z_{i}$ we find that they are identically zero.

Now, we can write

$$
\phi_{p}^{(1)}=\prod_{i=1}^{2 n-2}\left(z_{i+2}-q^{2} z_{i}\right) \phi_{p}^{(2)}
$$

where $\phi_{p}^{(2)}$ has degree $\delta_{1}=\delta_{2 p}=p-3, \delta_{2}=\delta_{2 p-1}=p-4$ and all the others $\delta_{i}=p-5$.

Clearly, this procedure can be repeated; at step $r, \phi_{p}^{(r)}$ has degree:

$$
\begin{aligned}
\delta_{1} & =p-r-1 \\
\delta_{2} & =p-r-2 \\
\vdots & \\
\delta_{r} & =p-2 r \\
\vdots & \\
\delta_{i} & =p-2 r-1 \\
\vdots & \\
\delta_{2 p} & =p-r-1
\end{aligned}
$$

We write

$$
\phi_{p \mid z_{i+r+1}=q^{2} z_{i}}^{(r)}=\prod_{j \notin[i-r, i+2 r+1]}\left(q z_{i}-z_{j}\right) \psi_{p}^{(r, i)}
$$


Counting the degree in $z_{i}$ we conclude that $\psi_{p}^{(r, i)}=0$. So we can construct $\phi_{p}^{(r+1)}$.

When $r \geq \frac{n}{2}$ we obtain a polynomial of negative degree which implies that the polynomial is identically zero.

Remark: What this lemma shows in other words is that the vector space of polynomials of degree at most $n-1$ in each variable satisfying the wheel condition is of dimension at most $c_{n}$. In fact it is known to be of dimension exactly $c_{n}$; the standard proof involves the fact that it is an irreducible representation of the affine Hecke algebra, see e.g. [18, 9].

\section{An antisymmetrization formula}

The goal of this section is to prove identity (4.16), which allows to turn an equation of the type (4.15) into one of the type (4.9). Identity (4.16) was conjectured by Di Francesco and Zinn-Justin in [6] and proved by Zeilberger [24]. Equivalently, it was proved that the integrand of the 1.h.s. without the factor $\varphi(u)$, once antisymmetrized and truncated to its negative degree part (the positive powers of the $u_{i}$ cannot contribute to the integral), reduces to the integrand of the r.h.s. without the factor $\varphi(u)$. Here we prove in an independent way a much stronger statement. Indeed, here we perform the exact antisymmetrization of a spectral parameter dependent generalization of the integrand. ${ }^{2}$

\section{D.1 The general case}

Let $h_{q}(x, y)=\left(q x-q^{-1} y\right)\left(q x y-q^{-1}\right)\left(\right.$ and, obviously, $\left.h_{1}(x, y)=(x-y)(x y-1)\right)$. Let us also define

$$
\begin{aligned}
f(w, z) & =\frac{1}{z\left(1-q^{2} w^{2}\right)\left(q^{-2}-1\right)}\left(\frac{1}{h_{1}(w, z)}-\frac{1}{h_{q}(w, z)}\right) \\
& =\frac{1}{h_{1}(w, z) h_{q}(w, z)}
\end{aligned}
$$

The quantity of interest is

$$
B_{n}(w, z)=A S\left\{\frac{\prod_{i<j}\left(q w_{i}-q^{-1} w_{j}\right)}{\prod_{i \leq j} h_{1}\left(w_{j}, z_{i}\right) \prod_{i \geq j} h_{q}\left(w_{j}, z_{i}\right)}\right\}
$$

\footnotetext{
${ }^{2}$ More precisely, the expression we antisymmetrize is the integrand before the homogeneous limit in which spectral parameters come in pairs $\{z, 1 / z\}$.
} 
where $A S(\phi)\left(w_{1}, \ldots, w_{n}\right)=\sum_{\sigma \in \mathcal{S}_{n}}(-1)^{|\sigma|} \phi\left(w_{\sigma(1)}, \ldots, w_{\sigma(n)}\right)$

We then claim that $B_{n}$ can be written as:

$$
B_{n}(w, z)=\frac{q^{\frac{n(n-1)}{2}} \mathbf{f}_{n}}{\prod_{i<j} h_{1}\left(z_{i}, z_{j}\right)\left(1-q^{2} w_{i} w_{j}\right)}
$$

where $\mathbf{f}_{n}=\operatorname{det}\left[f\left(w_{i}, z_{j}\right)\right]_{i, j \leq n}$.

Again, we prove it by induction. For $n=1$, we obtain on both sides:

$$
B_{1}=\frac{1}{h_{1}\left(w_{1}, z_{1}\right) h_{q}\left(w_{1}, z_{1}\right)}
$$

Let the equality of (D.2) and (D.3) hold at $n-1$. Starting from (D.2) and pushing $z_{n}$ and $w_{j}$ out of the anti-symmetrization we can write our equation as follows:

$B_{n}(w, z)=\sum_{j}(-1)^{n+j} \frac{\prod_{i \neq j}\left(q w_{i}-q^{-1} w_{j}\right)}{\prod_{i} h_{1}\left(w_{j}, z_{i}\right) h_{q}\left(w_{i}, z_{n}\right)} A S\left\{\frac{\prod_{i<k}\left(q w_{l}-q^{-1} w_{k}\right)}{\prod_{i \leq k} h_{1}\left(w_{k}, z_{i}\right) \prod_{i \geq k} h_{q}\left(w_{k}, z_{i}\right)}\right\}_{\hat{z}_{n} \hat{w}_{j}}$

where the hat over $\hat{z_{n}}$ and $\hat{w}_{j}$ means that the terms that include them are absent from the anti-symmetrization. We use the hypothesis to replace the anti-symmetrization part:

$$
\begin{aligned}
B_{n} & =\sum_{j}(-1)^{n+j} \frac{\prod_{i \neq j}\left(q w_{i}-q^{-1} w_{j}\right)}{\prod_{i} h_{1}\left(w_{j}, z_{i}\right) h_{q}\left(w_{i}, z_{n}\right)} \frac{q^{\frac{(n-1)(n-2)}{2}} \mathbf{f}_{n-1, \hat{w}_{j} \hat{z}_{n}}}{\left(\prod_{i<k} h_{1}\left(z_{i}, z_{k}\right)\left(1-q^{2} w_{i} w_{k}\right)\right)_{\hat{w}_{j} \hat{z}_{n}}} \\
& =\sum_{j}(-1)^{n+j} \frac{\prod_{i \neq j} h_{q}\left(w_{i}, w_{j}\right) \prod_{i \neq n} h_{1}\left(z_{i}, z_{n}\right)}{\prod_{i} h_{1}\left(w_{j}, z_{i}\right) h_{q}\left(w_{i}, z_{n}\right)} \frac{(-1)^{n-1} q^{\frac{n(n-1)}{2}} \mathbf{f}_{n-1, \hat{w}_{j} \hat{z}_{n}}}{\left(\prod_{i<k} h_{1}\left(z_{i}, z_{k}\right)\left(1-q^{2} w_{i} w_{k}\right)\right)}
\end{aligned}
$$

The idea now is to rewrite this under the form $\sum_{j}(-1)^{n+j} \mathbf{f}_{n-1, \hat{w}_{j} \hat{z}_{n}} \sum_{i} g_{i} f\left(w_{j}, z_{i}\right)$ for some functions $g_{i}$. Indeed, using the fact that $\mathbf{f}_{n}$ is a determinant, we would get

$$
\sum_{j}(-1)^{n+j} \mathbf{f}_{n-1, \hat{w}_{j} \hat{z}_{n}} \sum_{i} g_{i} f\left(w_{j}, z_{i}\right)=\sum_{j}(-1)^{n+j} \mathbf{f}_{n-1, \hat{w}_{j} \hat{z}_{n}} g_{n} f\left(w_{j}, z_{n}\right)=\mathbf{f}_{n} g_{n}
$$

One can guess the form of $g_{i}$ :

$$
g_{i}=\frac{\prod_{j \neq i, n} h_{1}\left(z_{j}, z_{n}\right) \prod_{j} h_{q}\left(w_{j}, z_{i}\right)}{\prod_{j \neq i, n} h_{1}\left(z_{i}, z_{j}\right) \prod_{j} h_{q}\left(w_{j}, z_{n}\right)}
$$


One can verify this decomposition directly. Equivalently, it can be written as

$$
\sum_{i} \frac{\prod_{j \neq i, n} h_{1}\left(z_{j}, z_{n}\right) \prod_{j} h_{q}\left(w_{j}, z_{i}\right)}{\prod_{j \neq i, n} h_{1}\left(z_{i}, z_{j}\right) \prod_{j} h_{q}\left(w_{j}, z_{n}\right)} f\left(w_{k}, z_{i}\right)=\frac{\prod_{i \neq k} h_{q}\left(w_{i}, w_{k}\right) \prod_{i \neq n} h_{1}\left(z_{i}, z_{n}\right)}{\prod_{i} h_{1}\left(w_{k}, z_{i}\right) h_{q}\left(w_{i}, z_{n}\right)}
$$

or, by multiply both sides with $\prod_{i} h_{1}\left(w_{k}, z_{i}\right) h_{q}\left(w_{i}, z_{n}\right)$ to obtain polynomials of $w_{k}$ of degree $2(n-1)$ :

$$
\sum_{i} \frac{\prod_{j \neq i, n} h_{1}\left(z_{j}, z_{n}\right)}{\prod_{j \neq i, n} h_{1}\left(z_{i}, z_{j}\right)} \prod_{j \neq k} h_{q}\left(w_{j}, z_{i}\right) \prod_{j \neq i} h_{1}\left(w_{k}, z_{j}\right)=\prod_{i \neq k} h_{q}\left(w_{i}, w_{k}\right) \prod_{i \neq n} h_{1}\left(z_{i}, z_{n}\right)
$$

It is enough to prove that this equation is the same in all points $w_{k}=z_{i}$ and $w_{k}=z_{i}^{-1}$. In the first case we have:

$$
\begin{aligned}
\frac{\prod_{j \neq i, n} h_{1}\left(z_{j}, z_{n}\right)}{\prod_{j \neq i, n} h_{1}\left(z_{i}, z_{j}\right)} \prod_{j \neq k} h_{q}\left(w_{j}, z_{i}\right) \prod_{j \neq i} h_{1}\left(z_{i}, z_{j}\right) & =\prod_{j \neq k} h_{q}\left(w_{j}, z_{i}\right) \prod_{j \neq n} h_{1}\left(z_{j}, z_{n}\right) \\
\prod_{j \neq i, n} h_{1}\left(z_{j}, z_{n}\right) \prod_{j \neq i} h_{1}\left(z_{i}, z_{j}\right) & =\prod_{j \neq n} h_{1}\left(z_{j}, z_{n}\right) \prod_{j \neq i, n} h_{1}\left(z_{i}, z_{j}\right)
\end{aligned}
$$

which is always true. In the second case $w_{k}=z_{i}^{-1}$ :

$$
\frac{\prod_{j \neq i, n} h_{1}\left(z_{j}, z_{n}\right)}{\prod_{j \neq i, n} h_{1}\left(z_{i}, z_{j}\right)} \prod_{j \neq k} h_{q}\left(w_{j}, z_{i}\right) \prod_{j \neq i} h_{1}\left(z_{i}^{-1}, z_{j}\right)=\prod_{j \neq k} h_{q}\left(w_{j}, z_{i}^{-1}\right) \prod_{j \neq n} h_{1}\left(z_{i}, z_{n}\right)
$$

multiplying both sides by $z_{i}^{2(n-1)}$ and knowing that $z_{i}^{2} h_{1}\left(z_{i}^{-1}, x\right)=h_{1}\left(z_{i}, x\right)$ and $z_{i}^{2} h_{q}\left(x, z_{i}^{-1}\right)=h_{q}\left(x, z_{i}\right)$ we obtain the same equality.

Finally we calculate $g_{n}$ :

$$
g_{n}=\frac{\prod_{j \neq n} h_{1}\left(z_{j}, z_{n}\right) \prod_{j} h_{q}\left(w_{j}, z_{n}\right)}{\prod_{j \neq n} h_{1}\left(z_{n}, z_{j}\right) \prod_{j} h_{q}\left(w_{j}, z_{n}\right)}=(-1)^{n-1}
$$

we replace $\sum_{i} g_{i} f\left(w_{j}, z_{i}\right)$ by $g_{n} f\left(w_{j}, z_{n}\right)$ :

$$
\begin{aligned}
B_{n} & =\sum_{j}(-1)^{n+j} \frac{q^{n(n-1) / 2} \mathbf{f}_{n-1, \hat{w}_{j} \hat{z}_{n}} f\left(w_{j}, z_{n}\right)}{\left(\prod_{i<k} h_{1}\left(z_{i}, z_{k}\right)\left(1-q^{2} w_{i} w_{k}\right)\right)} \\
& =q^{\frac{n(n-1)}{2}} \frac{\mathbf{f}_{n}}{\left(\prod_{i<k} h_{1}\left(z_{i}, z_{k}\right)\left(1-q^{2} w_{i} w_{k}\right)\right)}
\end{aligned}
$$




\section{D.2 Integral version}

A special case (of direct interest to us) is when we integrate $B_{n}$ on a contour which surrounds only the poles $w_{i}=z_{j}^{ \pm 1}$. Let us thus consider the following integral

$$
\oint \ldots \oint \prod_{i} \frac{d w_{i}}{2 \pi i} \psi(w, z) B_{n}(w, z)
$$

where $\psi(w, z)$ is an analytic function of the $w$ in the integration region. Looking at the expression (D.1), we note that if in the calculation of $\mathbf{f}_{n}$ we pick a term with at least one $h_{q}\left(w_{i}, z_{j}\right)$ there will be fewer than $n$ poles and the integral will be zero. This way, we can erase all the terms with $h_{q}\left(w_{i}, z_{j}\right)$, and form the restricted $\overline{\mathbf{f}}_{n}$ :

$$
\overline{\mathbf{f}}_{n}=\frac{1}{\left(q^{-2}-1\right)^{n} \prod_{i} z_{i}\left(1-q^{2} w_{i}^{2}\right)} \operatorname{det}\left|\frac{1}{h_{1}\left(w_{i}, z_{j}\right)}\right|
$$

If we rewrite $h_{1}\left(w_{i}, z_{j}\right)=w_{i} z_{j}\left(w_{i}+w_{i}^{-1}-z_{j}-z_{j}^{-1}\right)$ we easily identify $\overline{\mathbf{f}}$ with a Cauchy determinant, which can be evaluated:

$$
\begin{aligned}
\overline{\mathbf{f}}_{n} & =\frac{\prod_{i<j}\left(w_{i}+w_{i}^{-1}-w_{j}-w_{j}^{-1}\right)\left(z_{j}+z_{j}^{-1}-z_{i}-z_{i}^{-1}\right)}{\left(q^{-2}-1\right)^{n} \prod_{i} z_{i}^{2} w_{i}\left(1-q^{2} w_{i}^{2}\right) \prod_{i, j}\left(w_{i}+w_{i}^{-1}-z_{j}-z_{j}^{-1}\right)} \\
& =\frac{1}{\left(q^{-2}-1\right)^{n} \prod_{i} z_{i}\left(1-q^{2} w_{i}^{2}\right)} \frac{\prod_{i<j} h_{1}\left(w_{i}, w_{j}\right) h_{1}\left(z_{j}, z_{i}\right)}{\prod_{i, j} h_{1}\left(w_{i}, z_{j}\right)}
\end{aligned}
$$

Thus, in Eq. (D.9) one can rewrite $B_{n}$, given in general by Eq. (D.3), as the same expression in which $\mathbf{f}_{n}$ is replaced with $\overline{\mathbf{f}}_{n}$.

Let us now assume that $\psi$ is of the form $\psi(w, z)=\prod_{i<j}\left(w_{j}-w_{i}\right) \phi(w, z)$ where $\phi$ is symmetric in the $w_{i}$. Then

$$
\begin{aligned}
& \oint \ldots \oint \prod_{i} \frac{d w_{i}}{2 \pi i} \psi(w, z) B_{n}(w, z) \\
& \quad=n ! \oint \ldots \oint \prod_{i} \frac{d w_{i}}{2 \pi i} \phi(w, z) \frac{\prod_{i<j}\left(w_{j}-w_{i}\right)\left(q w_{i}-q^{-1} w_{j}\right)}{\prod_{i \leq j} h_{1}\left(w_{j}, z_{i}\right) \prod_{i \geq j} h_{q}\left(w_{j}, z_{i}\right)} \\
& =\frac{1}{\left(q^{-2}-1\right)^{n}} \oint \ldots \oint \prod_{i} \frac{d w_{i}}{z_{i} 2 \pi i} \phi(w, z) \frac{q^{\frac{n(n-1)}{2}} \prod_{i<j}\left(w_{j}-w_{i}\right) h_{1}\left(w_{j}, w_{i}\right)}{\prod_{i \leq j}\left(1-q^{2} w_{i} w_{j}\right) \prod_{i, j} h_{1}\left(w_{i}, z_{j}\right)}
\end{aligned}
$$




\section{D.3 Homogeneous Limit}

The case of interest to us is when we set all the $z_{i}=1$. We can then use the same transformation as before:

$$
u_{i}=\frac{w_{i}-1}{q w_{i}-q^{-1}}
$$

to deduce the desired equation from Eq. (D.10).

Call $\varphi(u)=\prod_{i}\left(1-q u_{i}\right)^{2} \phi\left(w_{i}=\frac{1-q^{-1} u_{i}}{1-q u_{i}}, z_{i}=1\right)$. The second line becomes

$$
n ! \oint \ldots \oint \prod_{i} \frac{d u_{i}}{2 \pi i u_{i}^{2 i}} \frac{\varphi(u)}{\left(q-q^{-1}\right)^{n(n+2)}} \prod_{i<j}\left(u_{j}-u_{i}\right)\left(1+\tau u_{j}+u_{i} u_{j}\right)
$$

while the expression on the third line becomes

$$
\oint \ldots \oint \prod_{i} \frac{d u_{i}}{2 \pi i u_{i}^{2 n}} \frac{\varphi(u)}{\left(q-q^{-1}\right)^{n(n+2)}} \frac{\prod_{i<j}\left(u_{j}-u_{i}\right)\left(u_{i}-u_{j}\right)\left(u_{i}+u_{i}+\tau u_{i} u_{j}\right)}{\prod_{i \leq j}\left(1-u_{i} u_{j}\right)}
$$

In both cases, the integrals surround zero.

In the latter, one can reinterpret some factors as a Vandermonde determinant:

$$
A S\left\{\prod_{i} \frac{\left(1+\tau u_{i}\right)^{i-1}}{u_{i}^{2 i}}\right\}=\prod_{i} \frac{1}{u_{i}^{2 n}} \prod_{i<j}\left(u_{i}-u_{j}\right)\left(u_{i}+u_{j}+\tau u_{i} u_{j}\right)
$$

and replace to obtain our final result:

$$
\begin{aligned}
\oint \ldots \oint \prod_{i} \frac{d u_{i}}{2 \pi i} \frac{\varphi(u)}{u_{i}^{2 i}} \prod_{i<j}\left(u_{j}-u_{i}\right)\left(1+\tau u_{j}+u_{i} u_{j}\right) \\
\quad=\oint \ldots \oint \prod_{i} \frac{d u_{i}}{2 \pi i} \varphi(u) \frac{\left(1+\tau u_{i}\right)^{i-1}}{u_{i}^{2 i}} \frac{\prod_{i<j}\left(u_{j}-u_{i}\right)}{\prod_{i \leq j}\left(1-u_{i} u_{j}\right)}
\end{aligned}
$$

where we recall that $\varphi(u)$ is some analytic function in a neighborhood of zero (that is, without poles in this domain) and symmetric in the $u_{i}$.

\section{References}

[1] M. T. Batchelor, J. de Gier, and B. Nienhuis, The quantum symmetric XXZ chain at $\Delta=-1 / 2$, alternating sign matrices and plane partitions, J. Phys. A 34 (2001), L265, arXiv: cond-mat/0101385. 
[2] David M. Bressoud, Proofs and confirmations, MAA Spectrum, Mathematical Association of America, Washington, DC, 1999, The story of the alternating sign matrix conjecture. MR1718370 (2000i:15002)

[3] Jan de Gier, Loops, matchings and alternating-sign matrices, Discr. Math. 298 (2005), 365, arXiv:math/0211285.

[4] P. Di Francesco and P. Zinn-Justin, Around the Razumov-Stroganov conjecture: Proof of a multi-parameter sum rule, E. J. Combi. 12 (2005), no. 1, R6, arXiv:math-ph/0410061.

[5] —, Quantum Knizhnik-Zamolodchikov equation, generalized RazumovStroganov sum rules and extended Joseph polynomials, J. Phys. A 38 (2005), L815-L822, arXiv:math-ph/0508059.

[6] __, Quantum Knizhnik-Zamolodchikov equation, totally symmetric selfcomplementary plane partitions and alternating sign matrices, Theor. and Math. Phys. (2008), arXiv:math-ph/0703015.

[7] Ira Gessel and Gérard Viennot, Binomial determinants, paths, and hook length formulae, Advances in mathematics 58 (1985), no. 3, 300-321.

[8] A. G. Izergin, Partition function of a six-vertex model in a finite volume, Dokl. Akad. Nauk SSSR 297 (1987), no. 2, 331-333. MR919260 (88m:82040)

[9] M. Kasatani, Subrepresentations in the polynomial representation of the double affine Hecke algebra of type $G L_{n}$ at $t^{k+1} q^{r-1}=1$, Int. Math. Res. Not. 28 (2005), 1717-1742, arXiv:math/0501272.

[10] V. Korepin and P. Zinn-Justin, Thermodynamic limit of the six-vertex model with domain wall boundary conditions, J. Phys. A 40 (2000), 7053, arXiv: cond-mat/0004250.

[11] V. E. Korepin, Calculation of norms of Bethe wave functions, Comm. Math. Phys. 86 (1982), no. 3, 391-418. MR677006 (83m:81078)

[12] Greg Kuperberg, Another proof of the alternating-sign matrix conjecture, Internat. Math. Res. Notices (1996), no. 3, 139-150, arXiv:math/9712207.

[13] Bernt Lindström, On the vector representations of induced matroids, Bull. London Math. Soc. 5 (1973), 85-90. MR0335313 (49 \#95)

[14] W. H. Mills, David P. Robbins, and Howard Rumsey, Jr., Proof of the Macdonald conjecture, Invent. Math. 66 (1982), no. 1, 73-87. MR652647 (83i:05013)

[15] — Alternating sign matrices and descending plane partitions, J. Combin. Theory Ser. A 34 (1983), no. 3, 340-359. MR700040 (85b:05013) 
[16] _ Self-complementary totally symmetric plane partitions, J. Combin. Theory Ser. A 42 (1986), no. 2, 277-292. MR847558 (88b:05008)

[17] Soichi Okada, Enumeration of symmetry classes of alternating sign matrices and characters of classical groups, J. Algebraic Combin. 23 (2006), no. 1, 4369, arXiv:math/0408234, doi:10.1007/s10801-006-6028-3.

[18] V. Pasquier, Quantum incompressibility and Razumov Stroganov type conjectures, Ann. Henri Poincaré 7 (2006), 397-421, arXiv: cond-mat/0506075.

[19] A. V. Razumov and Yu G. Stroganov, Combinatorial nature of ground state vector of $O(1)$ loop model, Teor. Mat. Fiz. 138 (2004), 395, arXiv:math/0104216.

[20] David P. Robbins, The story of 1,2,7,42, 429, 7436,..., Math. Intelligencer 13 (1991), no. 2, 12-19. MR1098216 (92d:05010)

[21] David P. Robbins and Howard Rumsey, Jr., Determinants and alternating sign matrices, Adv. in Math. 62 (1986), no. 2, 169-184. MR865837 (88a:15012)

[22] Yu. Stroganov, Izergin-Korepin determinant at a third root of unity, Teor. Mat. Fiz. 1 (2006), 65-76, arXiv:math-ph/0204042.

[23] Doron Zeilberger, Proof of the alternating sign matrix conjecture, Electron. J. Combin. 3 (1996), no. 2, Research Paper 13, approx. 84 pp. (electronic), The Foata Festschrift, arXiv:math/9407211.

[24] Doron Zeilberger, Proof of a conjecture of Philippe Di Francesco and Paul Zinn-Justin related to the $q K Z$ equations and to Dave Robbins' two favorite combinatorial objects, 2007, http://www.math.rutgers.edu $/ \sim$ zeilberg/mamarim/mamarimhtml/diFrancesco.html. 